\title{
CHROMOSOME NUMBERS OF FLOWERING PLANTS IN THE NETHERLANDS
}

\author{
TH. W. J. GADELLA and E. KLIPHUIS \\ (Botanical Museum and Herbarium, Utrecht) \\ (received February 8th, 1963)
}

\begin{abstract}
154 plant species, chosen at random, and collected in the Netherlands were investigated cytologically. The chromosome numbers determined were compared with data known from other countries.
\end{abstract}

\section{INTRODUCTION}

Up to now different lists and atlasses with chromosome numbers of plants were published, mostly concerning distinct areas as: The Alps, (FAvager, 1949, 1953, 1959); Central- and Northwest Europe (LöVE and Löve, 1961); England, (MAUde,-1939, 1940); Greenland, (Jörgensen, Sörensen and WestergaArd, 1958); Hungary, (Polya, 1949; Baksay, 1956); Iceland, (Löve and Löve, 1956); Poland, (Skalinska, 1950; Skalinska, et al., 1957, 1959); Romania, (TARNAVschI, 1948); Scandinavia, (Löve and Löve, 1942b); SchleswickHolstein, (WuLfF, 1937b+c, 1938, 1939, 1950).

The flora of the Netherlands has scarcely been investigated cytologically up to the present. Detailed cytological investigations have been done only on some genera. For lack of data chromosome numbers could not be listed before.

Cytogeographic investigations of different plant species have greatly contributed to a better understanding of the taxa concerned. So it seemed appropriate to initiate cytological research in the flora of the Netherlands. Therefore, in 1958, investigations were started for the preparation of a list of chromosome numbers. Plants of 154 species, chosen at random, all collected in the Netherlands, are treated in this article. It is the intention to extend this list in the future.

\section{Material AND METHODS}

Chromosome counts were made on metaphase plates of roottip cells. For this purpose roottips were fixed in Karpechenko. Fixations were made in the field directly, except for the genera Campanula, Galium and Viola, which were reared in pots in the open. The roottips were embedded in paraffin, sectioned at 15 micron and stained according to Heidenhain's haematoxylin method. Voucher specimens of all the investigated material have been deposited in the Botanical Museum and Herbarium, Utrecht.

\section{Results}

The results of the countings are given in the following tables: Table 1. Species with chromosome numbers in accordance with the literature.

Table 2. Species with intraspecific cytological variation.

Table 3. Species with chromosome numbers not determined before. 
Table 1. Chromosome numbers in accordance with the literature. In the first column the voucher number is listed, in the second the name of the species, in the third the somatic chromosome number, and in the fourth the origin of the material concerned.

Table 2. Species with intraspecific cytological variation. To the left the same grouping as in table one, to the right a survey of the cytological investigations up to the present. (In the first column the author and year of publication, in the second the origin and the chromosome number.)

Table 3. Species with chromosome numbers not determined before. Grouping of the table, the same as in table one.

TABLE I

Species with chromosome numbers in accordance with the literature.

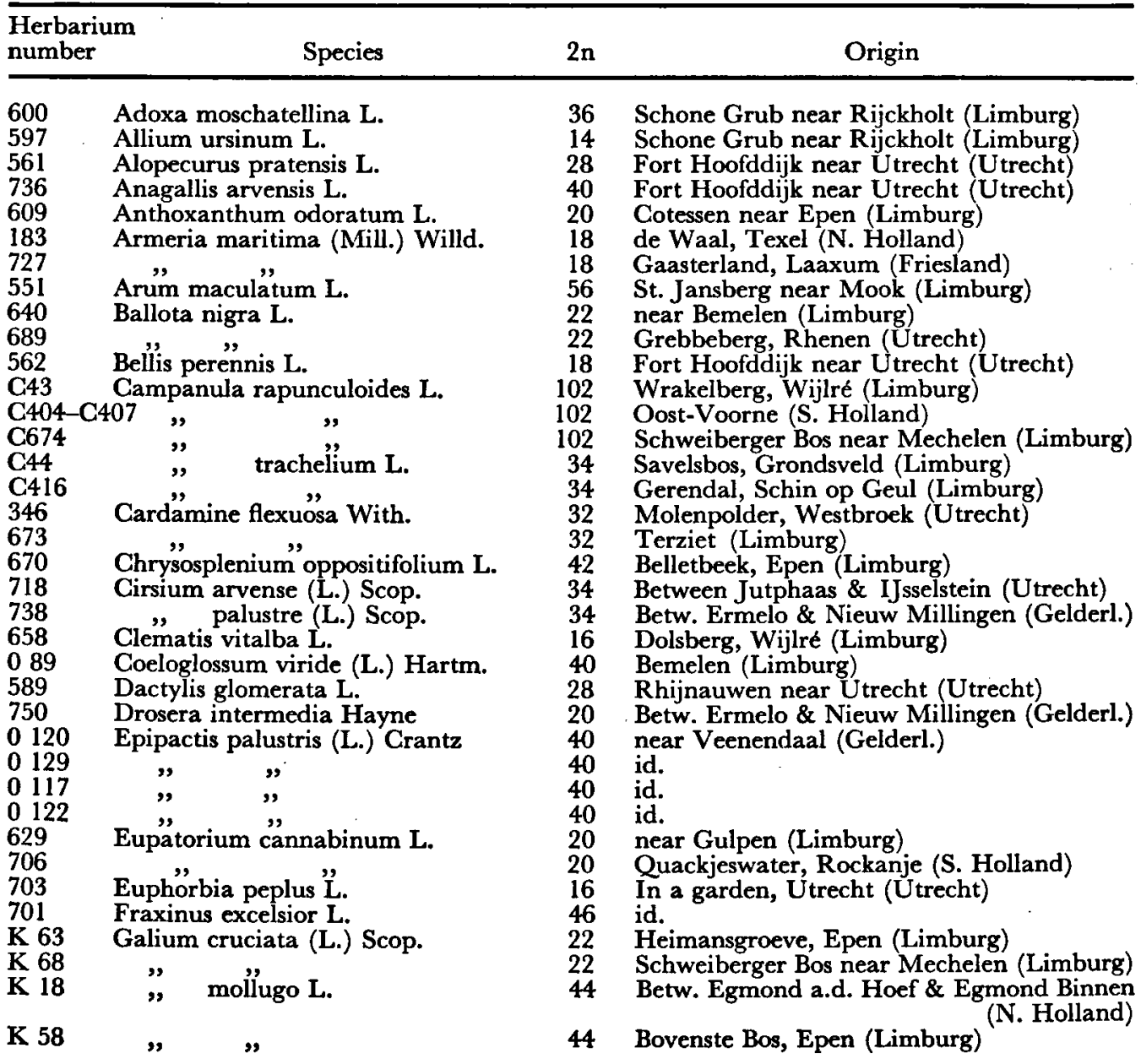


Table I (Cont.)

\begin{tabular}{|c|c|c|c|c|}
\hline & & Species & $2 n$ & Origin \\
\hline 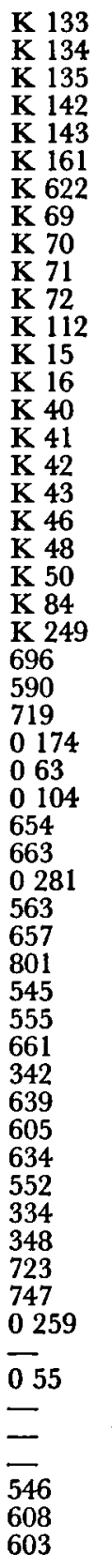 & 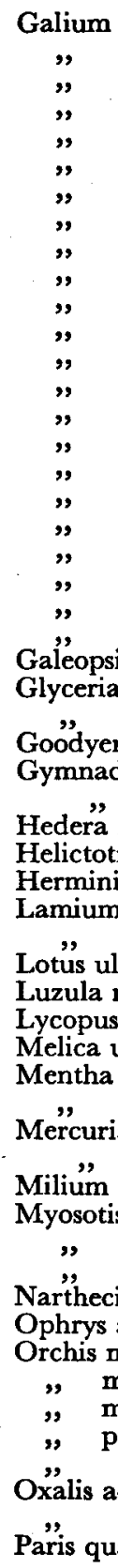 & 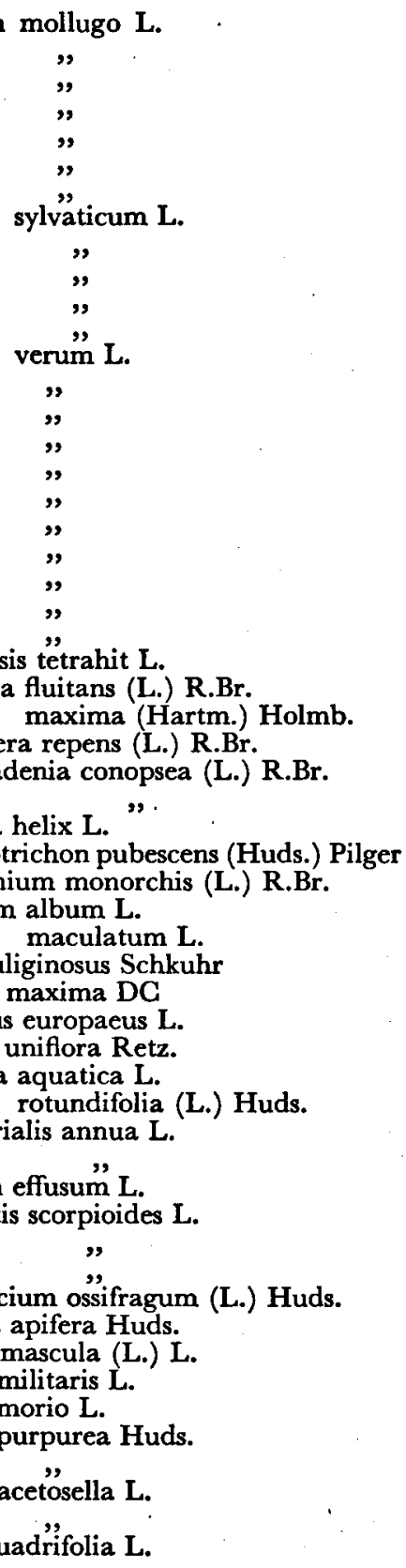 & $\begin{array}{l}44 \\
44 \\
44 \\
44 \\
44 \\
44 \\
44 \\
22 \\
22 \\
22 \\
22 \\
22 \\
44 \\
44 \\
44 \\
44 \\
44 \\
44 \\
44 \\
44 \\
44 \\
44 \\
44 \\
32 \\
40 \\
60 \\
40 \\
40 \\
40 \\
48 \\
14 \\
40 \\
18 \\
18 \\
12 \\
12 \\
22 \\
18 \\
96 \\
24 \\
16 \\
16 \\
28 \\
64 \\
64 \\
64 \\
26 \\
36 \\
42 \\
42 \\
36 \\
42 \\
42 \\
22 \\
22 \\
20\end{array}$ & $\begin{array}{l}\text { Weerdinge (Drenthe) } \\
\text { id. } \\
\text { id. } \\
\text { Johannapolder near Utrecht (Utrecht) } \\
\text { id. } \\
\text { Between Epen \& Mechelen (Limburg) } \\
\text { near Schalkwijk (Utrecht) } \\
\text { St. Jansberg near Mook (Limburg) } \\
\text { id. } \\
\text { id. } \\
\text { id. } \\
\text { id. } \\
\text { Between Bakkum \& Egmond (N. Holland) } \\
\text { id. } \\
\text { Kunrader Berg near Voerendaal (Limburg) } \\
\text { id. } \\
\text { id. } \\
\text { id. } \\
\text { id. } \\
\text { id. } \\
\text { id. } \\
\text { W. Kapelle (Zeeland) } \\
\text { Oude Mirdum (Friesland) } \\
\text { Grebbeberg, Rhenen (Utrecht) } \\
\text { Rhijnauwen near Utrecht (Utrecht) } \\
\text { near IJsselstein (Utrecht) } \\
\text { Schoorl (N. Holland) } \\
\text { Kunrader Berg near Voerendaal (Limburg) } \\
\text { Meeuwenkamp near Ede (Gelderl.) } \\
\text { Dolsberg, Wijlré (Limburg) } \\
\text { Gerendal, Schin op Geul (Limburg) } \\
\text { Schiermonnikoog (Friesland) } \\
\text { Fort Hoofddijk near Utrecht (Utrecht) } \\
\text { Between Eys \& Gulpen (Limburg) } \\
\text { Betw. Ermelo \& Nieuw Millingen (Gelderl.) } \\
\text { St. Jansberg, Mook (Limburg) } \\
\text { id. } \\
\text { Gerendal, Schin op Geul (Limburg) } \\
\text { Molenpolder, Westbroek (Utrecht) } \\
\text { Bemelerberg, Bemelen (Limburg) } \\
\text { near Rijckholt (Limburg) } \\
\text { Kadier en Keer (Limburg) } \\
\text { St. Jansberg, Mook (Limburg) } \\
\text { Molenpolder, Westbroek (Utrecht) } \\
\text { Molenpolder, Westbroek (Utrecht) } \\
\text { near Jutphaas (Utrecht) } \\
\text { Betw. Ermelo \& Nieuw Millingen (Gelderl.) } \\
\text { Wrakelberg, Wijlré (Limburg) } \\
\text { Bovenste Bos, Epen (Limburg) } \\
\text { Savelsbos, Grondsveld (Limburg) } \\
\text { Rozenkoele, Kadier en Keer (Limburg) } \\
\text { Geerendal, Schin op Geul (Limburg) } \\
\text { Schweiberger Bos near Mechelen (Limburg) } \\
\text { St. Jansberg near Mook (Limburg) } \\
\text { Bovenste Bos, Epen (Limburg) } \\
\text { Schone Grub, Rijckholt (Limburg) } \\
\text { idimb }\end{array}$ \\
\hline
\end{tabular}


Table I (Cont.)

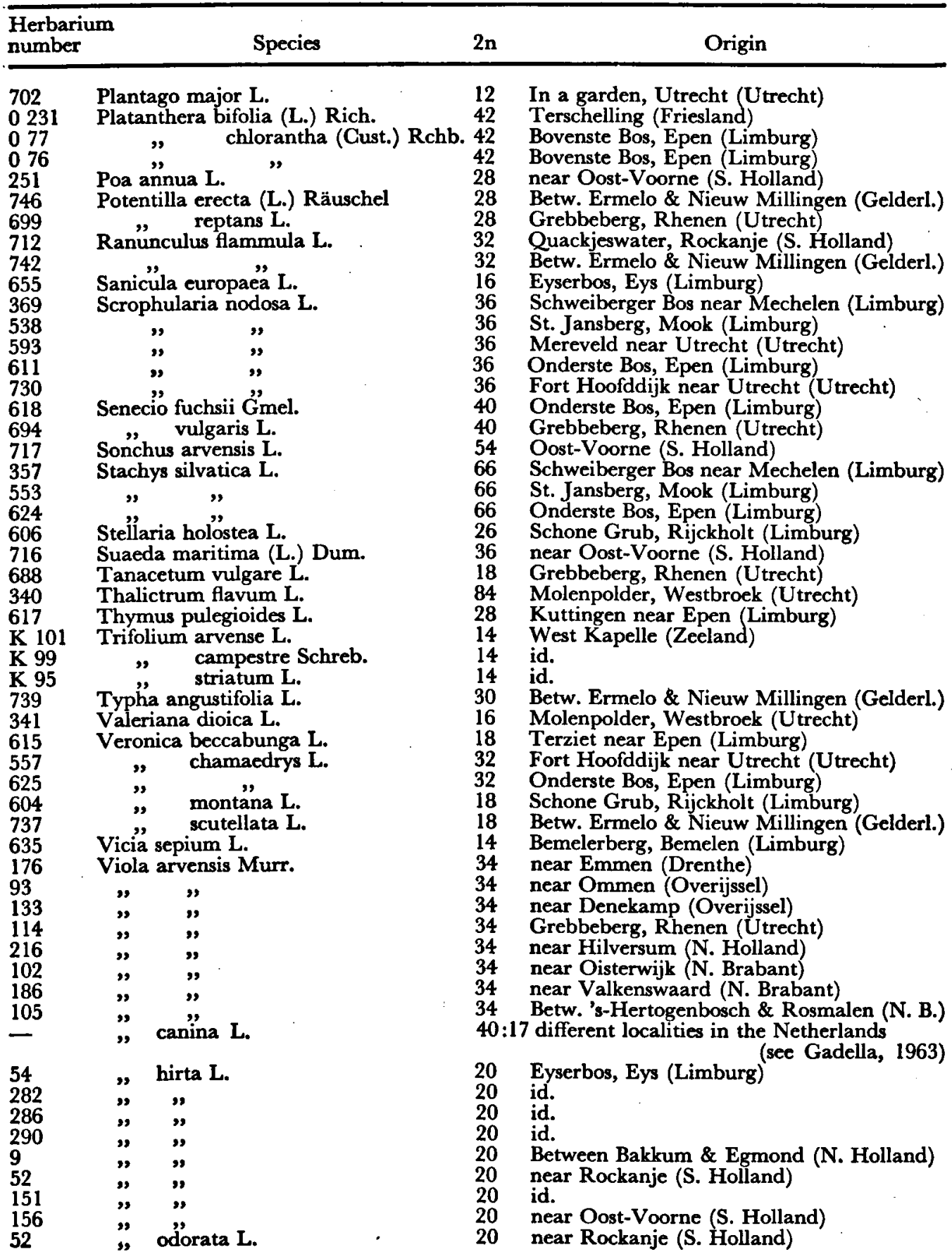


Table 1 (Cont.)

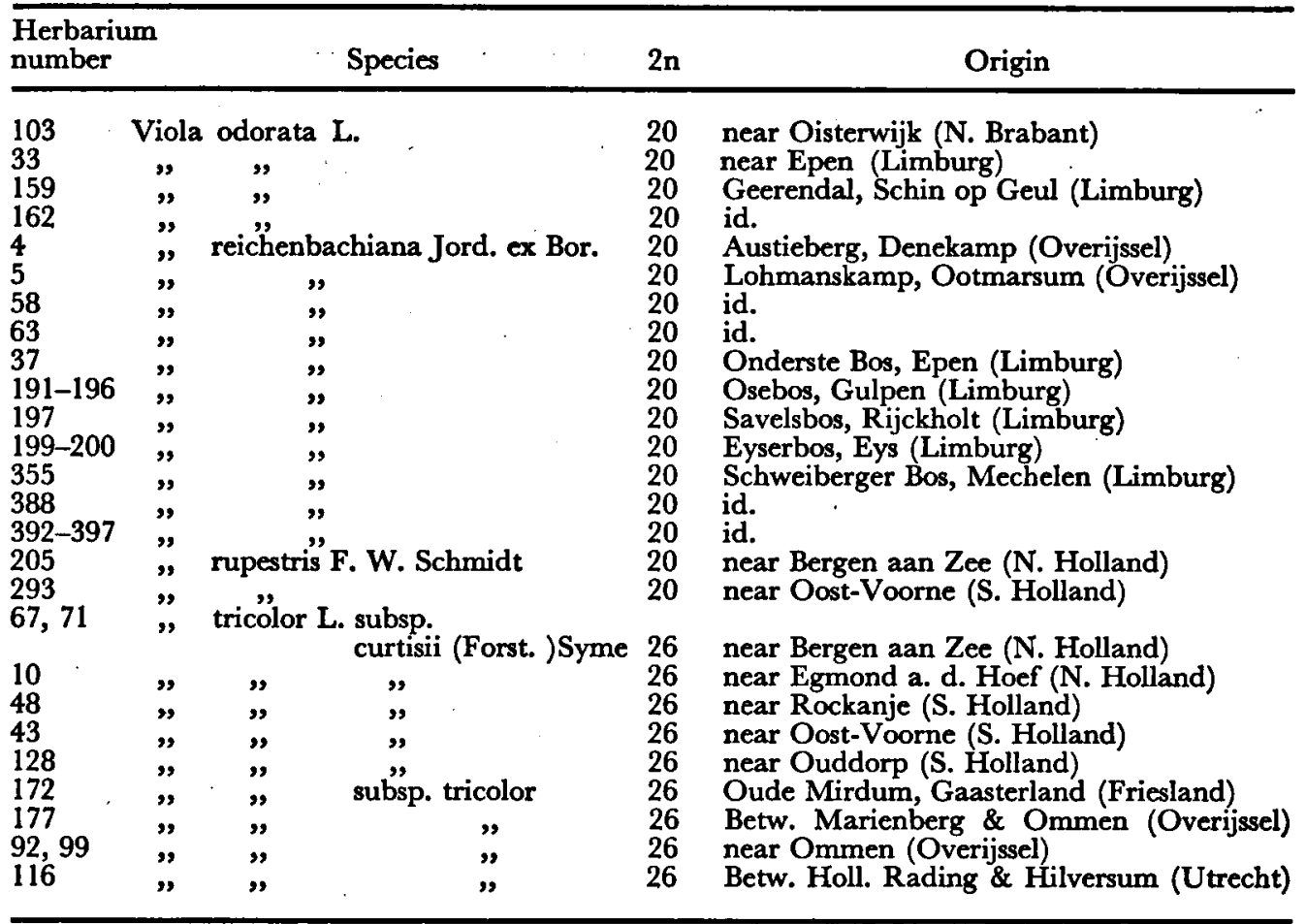


TH. W. J. GADELla AND E. KLIPHUTS

TABLE II

Species with intraspecific cytological variation

\begin{tabular}{rlll}
\hline Species & Coll. no. & Origin & 2n \\
\hline
\end{tabular}

Actaea spicata L.

659 Dolsberg near Wylré (Limburg)

16

Aethusa cynapium L.

$700 \quad$ Utrecht, in a garden (Utrecht)

20

Alisma plantago-aquatica L.

337

Molenpolder near Westbroek (Utrecht)

14

Alliaria petiolata

(Bieb.) Cavara et Grande

Artemisia vulgaris L.

525

636

687

668

Between Baarn and Eemdijk (Utrecht)

Between Houten and Schalkwijk (Utrecht)

Bemelerberg near Bemelen (Limburg)

Grebbeberg near Rhenen (Utrecht)

16

16

16

Butomus umbellatus L.
Between Wittem and Gulpen (Limburg)

St. Jansberg near Mook (Limburg)

36

Campanula rotundifolia L. (s.1.) C 39

near Maarn (Utrecht) 


\begin{tabular}{l}
\hline \multicolumn{1}{c}{ Author } \\
\hline Langlet (1927) \\
Polya (1950) \\
Rodriguez (1949) \\
Skalinska (in Skalinska et al., 1959) \\
Mattick (in Tischler, 1950)
\end{tabular}

Häkansson (1953)

Wanscher $(1931,1932)$

Schulz-Gaebel (1930)

Wewetzer (1956)

Wulff (1939)

Blomstrand (in Löve and Löve, 1944)

Löve and Löve (1942)

Palmgren (1943)

Tarnavschi (1948)

Björquist (1959); Löve and Löve (1942);

Erlandsson (1946)

de Castro and Noronha-Wagner (1950)

Erlandsson (1946)

Tschermak-Woess (1948)

Hendricks (1957)

Holzer (1952)

Pogan (1961)

Polya (1949)

Heppell (in Maude, 1939)

Priestly (1953)

Wulff (1950)

Baez Major (1934)

Winge (1932)

Jaretski (1932)

Polya (1949)

Clausen, Keck, Hiesey $(1939,1940)$

Keck (1946)

Löve and Löve (1956)

Mulligan (1957)

Polya (1949)

Wulff (1950); Khoshoo and Sobti (1958)

Khoshoo and Sobti (1958)

Harada (1948)

Harada (1956)

Lohammar (in Löve and Löve, 1948)

Löve and Löve (1961)

Rao (1953)

Skalinska (in Skalinska et al., 1961)

Whitaker (1934)

Lohammar (1931)

Lohammar (in Löve and Löve, 1948)

Lohammar (1931); Terby (1922)

Lohammar (1931)

Böcher (1936, 1960) unknown

Hungary

U.S.A.

Poland

Austria

Sweden

unknown

Germany

cult. mat.

Germany

Sweden

Sweden

Sweden

Romania

Sweden

Portugal

Denmark, Finland

Denmark

cult. mat.

Austria

Poland

Hungary

England

England

Germany

acc. to L. and L., 1961

unknown

unknown

Hungary

Sweden

North America

Iceland

Canada

Hungary

Germany

India

unknown

Japan

Scandinavia

Sweden, Canada

India

Poland

cult. mat.

Sweden

Scandinavia

Belgium

Germany, Sweden

Greenland
$2 n$

16

16

16

16

ca. 32

20

20

22

10

10

12

12

12

12

14

14

14

14

14

14

14

14

14

14

14,16

36

ca. $36-40$

ca. 42

42

16

16

16

16

16

16

36,54

26

26

26

26

26

26

26

28

39

40

40

34 
TH. W. J. GADELLA AND B. KLIPHUIS

TABLe II (Cont.)

\begin{tabular}{clll}
\hline \multicolumn{2}{c}{ Coll. no. } & \multicolumn{1}{c}{ Origin } & 2n \\
\hline Campanula rotundifolia L. (s.l.) & C 40 & near Valkenswaard (N. Brabant) & 68 \\
& C 71 & near Vledder (Drenthe) & 68 \\
& C 72 & Oude Mirdum, Gaasterland (Friesland) & 68 \\
& C 73 & Oude Mirdum, Gaasterland (Friesland) & 68 \\
& C 380 & Betw. Denekamp and Singraven (Overijss.) & 68 \\
& C 422 & near Marienberg (Overijssel) & 68 \\
& C 423 & Hoge Veluwe near Otterlo (Gelderl.) & 68 \\
& C 424 & Hoge Veluwe near Otterlo (Gelderl.) & 68 \\
& C 425 & Hoge Veluwe near Otterlo (Gelderl.) & 68 \\
& C 677 & St. Jansberg near Mook (Limburg) & 68 \\
& C 685 & Emmen (Drenthe) & 68
\end{tabular}

Cardamine amara L. $\quad 358$

Between Gulpen and Slenaken (Limburg)

Chrysanthemum leucanthemum 627

Between Gulpen and Mechelen (Limburg) L. (s.l.)

Comarum palustre L. 
Author

Böcher (1960)

Böcher and Larsen (1950)

Gadella (1962)

Böcher (1936, 1938)

Böcher (1960); Guinochet (1942); Gadella (1962)

Böcher (1938, 1960); Böcher and Larsen (1950)

Böcher (1936, 1960); Gadella (1962)

Böcher (1960); Gadella (1962)

Böcher (1960)

Gadella (1962)

Böcher and Larsen (1950); Löve and Löve (1956)

Hubac (1961); Gadella (1962)

Gadella (1962)

Banach (in Skalinska, 1950)

Lövkvist (1956, 1957)

Lövkvist (1957)

Manton (1932)

Lawrence (1932)

Lövkvist (1957); Mattick (in Tischler, 1950)

Baksay (1956, 1957); Polya (1950)

Böcher and Larsen (1957)

Cooper and Mahony (1935)

Czapick (in Skal. et al., 1961)

Dowrick (1952)

Ducker and Favarger (1956, 1957); Favarger (1959)

Martin and Smith (1955)

Böcher and Larsen (1957)

Czapick (in Skalinska et al., 1961)

Favarger (1959)

Löve and Löve (1956)

Mulligan (1958, 1959)

Mulligan (1958)

Polya (1950)

Rohweder (1937)

Shimotomai (1937)

Baksay (1956)

Böcher and Larsen (1957); Mulligan (1958)

Favarger (1959)

Czapick (in Skalinska et al., 1961)

Favarger (1959)

Ehrenberg (1945)

Löve and Löve (1956)

Wulff (1937c)

Jörgensen, et al. (1958)

Reese (in Löve and Löve, 1961)

Sokolovskaja and Strelkova (1941, 1960)

Sokolovskaja (1960)

Sokolovskaja-Kulczycka (in Skalinska et al. 1961)

Sokolovskaja and Strelkova (1960)

Palmgren (1943)
Origin

2n

France, Sweden, Denmark, U.S.S.R. 34

Greenland

Germany, Sweden, Austria

Greenland

France

Norway

Denmark

England, the Netherlands U.S.S.R., Germany, Sweden 68

Eire, Norway, Finland, Faroes, Yugoslavia

Czechoslovakia, Italy, Scotland 68

68

Iceland

France

Czecho-Slovakia

68

102

102

Poland

Sweden

Denmark, Ireland, France

England

England

Austria

16

16

16

16

32

32

Hungary

Poland, Denmark, England

18

North America

Poland

cult. mat.

Switzerland

U.S.A.

Sweden, Yugoslavia, Denmark,

France, U.S.S.R., Poland 36

Poland

Switzerland

Iceland

Canada

France, U.S.S.R.

Hungary

Germany

Austria

Hungary, Albania,

Portugal

Switzerland

Poland

Switzerland

18

18

18

18

18

18

36

36

36

36

36

36

36

36

54

54

a. 54

Scandinavia ' 28

Iceland 28

Germany : 28

Greenland $\quad 42$

Germany 42

U.S.S.R.

Sachalin $\quad 42$

Poland ' 42

U.S.S.R. $\quad 62-64$

Denmark 14 
TABle II (Gont.)

Species

Echinodorus ranunculoides

(L.) Engelm. ex Aschers.

Epipactis helleborine (L.) Crantz

Euphorbia cyparissias L.

Filipendula ulmaria (L.) Maxim.

725

Between Jutphaas and IJsselstein (Utrecht)

near Wageningen (Gelderland)

38

Emmerdennen near Emmen (Drenthe)

"Schovenhorst" Putten (Gelderland)

idem

38

38

537

St. Jansberg near Mook (Limburg)

20

14 and 16

Galium aparine $\mathbf{L}$.

K 33

K 34

K 17

K 248

K 435

K 436

K 191 near Weerdinge (Drenthe)

near Weerdinge (Drenthe)

near Putten (Gelderland)

Gaasterland, Oude Mirdum (Friesland)

Westenschouwen (Zeeland)

Westenschouwen (Zeeland)

near Putten (Gelderland)
66

64

64

64

63

63

66
Galium hercynicum Weig.

Galium palustre L.
K 252

K 364

K 386

K 414

K 39

K 154

K 155

K 381 near Duurswoude (Friesland)

near Oost-Voorne (S. Holland)

Eyserbos near Eys (Limburg)

near Duurswoude (Friesland)

Ravensbos near Valkenburg (Limburg)

Between Maarn and Maarsbergen (Utrecht)

idem

near Monnikendam (N. Holland)
44

44

44

44

24

24

24

24

Galium pumilum Murr. 


\section{Author}

Fernandes, Garcia and Fernandes (1948)

Löve and Löve (1944)

Packer (in Löve and Löve, 1961)

Hagerup (1944)

Weyer (1952)

Barber (1942)

Skalinska et al. (1957)

Hagerup (1945, 1947)

Skalinska et al. (1961)

Perry (1943)

Pritchard (1957, 1959)

Pritchard (1957, 1959); Shimoyama (1959)

Rutland (1941)

Moore (1958); Moore and Lindsay (1953)

Pritchard $(1957,1959)$

Wulff (1938)

Maude (1940)

Löve and Löve (1956)

Polya (1950)

Tarnavschi (1948)

Turesson (1938)

Piotrowicz (in Skalinska et al., 1959)

Kliphuis (1962)

Kliphuis (1962)

Böcher, Larsen and Rahn (1955)

Fagerlind (1934, 1937)

Kliphuis (1962)

Fagerlind (1934, 1937)

Kliphuis (1962)

Löve and Löve (1956)

Fagerlind (1934, 1937)

Kliphuis (1962)

Ehrendorfer (1956)

Fagerlind $(1934,1937)$

Kliphuis (1962)

Clapham (1949); Hancock (1942)

Fagerlind (1934, 1937)

Hagerup (1941)

Kliphuis (1962)

Piotrowicz (in Skalinska et al., 1961)

Clapham (1949)

Kliphuis (1962)

Clapman (1949); Hancock (1942)

Fagerlind (1934)

Kliphuis (1962)

Kliphuis (1962)

Piotrowicz (in Skalinska et al., 1959)

Ehrendorfer (1949)

$\begin{array}{ll}\text { Portugal } & 16 \\ \text { Sweden } & 16 \\ \text { Ireland } & 16 \\ \text { Denmark } & 18 \\ & \\ \text { the Netherlands } & 40(20) \\ \text { unknown } & 38 \\ \text { Poland } & 38 \\ \text { Denmark } & 38,40 \\ \text { Poland } & 40\end{array}$

U.S.A. $\quad 20$

France $\quad 20$

cult. mat. 20

England 20

Canada $\quad 40$

Great Britain $\quad 40$

Germany 14

cult. mat. 15

Iceland 16

Hungary 16

Romania 16

Siberia, Sweden $\quad 16$

Poland 42

Portugal, Canada 44

Denmark 63

Spain, France, Denmark, Sweden, Portugal

cult. mat. $\quad 64$

Austria, Denmark, France $\quad 64$

cult. mat. $\quad 66$

Portugal, France, Denmark, $\quad 66$

Iceland 66

$\begin{array}{ll}\text { cult. mat. } & 86,88\end{array}$

Portugal 22

Germany $\quad 44$

cult. mat. $\quad 44$

France, Germany 44

England 24

cult. mat. 24

Denmark 24

Germany $\quad 24$

Poland 24

England 48

Portugal 48

England $\quad 96$

cult. mat. $\quad 96$

Portugal 96

Denmark 44

Poland 44

Austria $\quad 66$ 
TABle II (Cont.)

$\begin{array}{llll}\text { Species } & \text { Coll. no. } & \text { Origin } & \text { 2n }\end{array}$

Galium pumilum Murr.

Galium uliginosum L.

Between Maarn and Maarsbergen (Utrecht)

22

K 190 Betw. Ermelo and Nw. Millingen (Gelderl.) idem

K $371 / 373$

K 32

K 153

near Oost-Voorne (S. Holland)

Weerdinge near Emmen (Drenthe)

K 188

Between Maarn and Maarsbergen (Utrecht) 44

Glechoma hederacea L. Betw. Ermelo and Nw. Millingen (Gelderl.) 44

Fort Hoofddijk near Utrecht (Utrecht)

St. Jansberg near Mook (Limburg)

Halimione portulacoides

(L.) Aellen

Hieracium pilosella $\mathbf{L}$.

Hippuris vulgaris $\mathbf{L}$.

Iris pseudacorus $\mathbf{L}$.

320

336
815 Boschplaat, Terschelling (Friesland)

36

586 Between Maarn and Maarsbergen (Utrecht)

36

711 Quackjeswater near Rockanje (S. Holland)

32

Rhijnauwen near Bunnik (Utrecht) Molenpolder near Westbroek (Utrecht)
Lamium galeobdolon (L.) L. $\quad 548$

Liparis loeselii (L.) Rich.
St. Jansberg near Mook (Limburg) Schone Grub near Rijckholt (Limburg) Onderste Bos near Epen (Limburg) 


\begin{tabular}{|c|c|c|}
\hline Author & Origin & $2 n$ \\
\hline $\begin{array}{l}\text { Ehrendorfer }(1949,1953) \\
\text { Ehrendorfer }(1955) \\
\text { Ehrendorfer }(1956) \\
\text { Goodway }(1955,1957) \\
\text { Kliphuis (1962) }\end{array}$ & $\begin{array}{l}\text { Austria } \\
\text { the Alps } \\
\text { Germany } \\
\text { England } \\
\text { Belgium }\end{array}$ & $\begin{array}{l}88 \\
88 \\
88 \\
88 \\
88\end{array}$ \\
\hline $\begin{array}{l}\text { Fagerlind (1934) } \\
\text { Hancock (1942) } \\
\text { Homeyer (1932) } \\
\text { Fagerlind (1934, 1937) } \\
\text { Löve and Löve (1956) }\end{array}$ & $\begin{array}{l}\text { cult. mat. } \\
\text { England } \\
\text { Germany } \\
\text { cult. mat. } \\
\text { Iceland }\end{array}$ & $\begin{array}{l}22 \\
22 \\
22 \\
44 \\
44\end{array}$ \\
\hline $\begin{array}{l}\text { Scheerer (1940) } \\
\text { Sugiura (1940) } \\
\text { Suzuka (1950) } \\
\text { Löve and Löve (1942) } \\
\text { Felföldy (1947); Polya (1949) } \\
\text { Hara (1954); Hara, Tanaka and Kurusawa (1954) } \\
\text { Löve and Löve (in Löve and Bernard, 1959) } \\
\text { Rutland (1941) } \\
\text { Skalinska (in Skalinska et al., 1959) }\end{array}$ & $\begin{array}{l}\text { Germany } \\
\text { cult. mat. } \\
\text { acc. to Löve and Love, } 1961 \\
\text { Sweden } \\
\text { Hungary } \\
\text { Japan } \\
\text { Canada } \\
\text { British Isles } \\
\text { Poland }\end{array}$ & $\begin{array}{l}18 \\
18 \\
18 \\
24 \\
36 \\
36 \\
36 \\
36 \\
36\end{array}$ \\
\hline $\begin{array}{l}\text { Tarnavschi (1948) } \\
\text { Castro and Fontes (1946) } \\
\text { Wulff (1937c) } \\
\text { Wulff }(1937 \mathrm{~b})\end{array}$ & $\begin{array}{l}\text { Romania } \\
\text { Portugal } \\
\text { Germany } \\
\text { unknown }\end{array}$ & $\begin{array}{l}18 \\
36 \\
36 \\
36\end{array}$ \\
\hline $\begin{array}{l}\text { Turesson and Turesson (1960) } \\
\text { Christoff and Popoff (1933) } \\
\text { Gentscheff (1937) } \\
\text { Turesson and Turesson (1960) }\end{array}$ & $\begin{array}{l}\text { Sweden } \\
\text { cult. mat. } \\
\text { cult. mat. } \\
\text { Sweden }\end{array}$ & $\begin{array}{l}36 \\
39,45 \\
45 \\
45,54,63\end{array}$ \\
\hline $\begin{array}{l}\text { Harada (1952) } \\
\text { Jörgensen, Sörensen and Westergaard (1958) } \\
\text { Löve and Löve (1956) } \\
\text { Sokolovskaja (1960) }\end{array}$ & $\begin{array}{l}\text { Japan } \\
\text { Greenland } \\
\text { Iceland } \\
\text { Sachalin }\end{array}$ & $\begin{array}{l}30 \\
32 \\
32 \\
32\end{array}$ \\
\hline $\begin{array}{l}\text { Wcislo (in Skalinska et al., 1957) } \\
\text { Polya (1949) } \\
\text { Heppell (in Maude, 1939) } \\
\text { Wcislo (in Skalinska et al., 1957) } \\
\text { Ehrenberg (1945) } \\
\text { Felföldy (1947); Polya (1949) } \\
\text { Fernandes, Garcia and Fernandes (1948) } \\
\text { Linder and Brun (1956); Simonet (1928) } \\
\text { Mori (1957) } \\
\text { Wcislo (in Skalinska et al., 1947) }\end{array}$ & $\begin{array}{l}\text { Poland } \\
\text { Hungary } \\
\text { England } \\
\text { Poland } \\
\text { Sweden } \\
\text { Hungary } \\
\text { Portugal } \\
\text { France } \\
\text { Italy } \\
\text { Poland }\end{array}$ & $\begin{array}{l}24 \\
24,30 \\
30 \\
32 \\
32-34 \\
34 \\
34 \\
34 \\
34 \\
34\end{array}$ \\
\hline $\begin{array}{l}\text { Polya }(1949) \\
\text { Turesson }(1938) \\
\text { Turesson }(1938) \\
\text { Turesson }(1938)\end{array}$ & $\begin{array}{l}\text { Hungary } \\
\text { Sweden } \\
\text { U.S.S.R. (Latvia) } \\
\text { Germany }\end{array}$ & $\begin{array}{l}18 \\
18 \\
18 \\
36\end{array}$ \\
\hline Hagerup (1941) & Denmark & 32 \\
\hline
\end{tabular}


TH. W. J. GADELla AND E. KLIPHUIS

TABle II (Cont.)

\begin{tabular}{lllc}
\hline \multicolumn{1}{c}{ Species } & \multicolumn{2}{c}{ Coll. no. } & Origin \\
\hline Listera cordata (L.) R.Br. & - & Schiermonnikoog (Friesland) & 40 \\
& & & \\
Listera ovata (L.) R.Br. & 0205 & Eyserbos near Eys (Limburg) & \\
& 0311 & near Oost-Voorne (S. Holland) & $35,35,36$ \\
& 0312 & near Oost-Voorne (S. Holland) & 37,38 \\
& 0110 & Allemanskamp near Veenendaal (Utrecht) & $34,35,38$ \\
& 0257 & Osebos near Gulpen (Limburg) & 34 \\
& 0111 & Allemanskamp near Veenendaal (Utrecht) & 36 \\
& 0313 & near Oost-Voorne (S. Holland) & 34
\end{tabular}

Lonicera periclymenum L. $\quad 534$

St. Jansberg near Mook (Limburg) near Vijlen (Limburg)

Quackjeswater near Rockanje (S. Holland)

Lysimachia nemorum L. $\quad 666$

Bunderbos near Bunde (Limburg)

Lysimachia nummularia L. $\quad 591$

Rhijnauwen near Bunnik (Utrecht)

Bemelen (Limburg)

Quackjeswater near Rockanje (S. Holland)

Fort Hoofddijk near Utrecht (Utrecht)

709 Quackjeswater near Rockanje (S. Holland) 56

Lysimachia vulgaris $\mathbf{L}$.

Mercurialis perennis $\mathbf{L}$.

Schweibergerbos near Mechelen (Limburg) 56

Myosotis sylvatica

355 Schweiberger Bos near Mechelen (Limburg)

Ehrh. ex Hoffm.

$$
\text { Schweiberger Bos near }
$$




\section{Author}

Origin

Iceland

Poland

U.S.S.R.

U.S.S.R.

unknown

Germany

Poland

unknown

Denmark

Sweden

Belgium

England

unknown

Great Britain

Denmark

England

unknown

France

Germany

unknown

Germany

unknown

28

Hungary

42

cult. mat.; Greece 18

Austria

Iceland

18

Geitler (1936); Griesinger (1937)

Löve and Löve (1956)

Geitler (1936); Griesinger (1937); Mattick (in Tischler, 1950)

Geitler (1936); Griesinger (1937)

Larsen (1960)

Rutland (1941)

Scheerer (1940)

de Barros Neves (1952, 1956)

idem

Czapick (in Skalinska et al., 1961)

Nakajima (1936); Rodriguez (1953); Sprumont (1928)

de Barros Neves (1952)

Polya (1950)

Sato (1942)

de Barros Neves (1952)

de Barros Neves (1952); Sprumont (1928)

de Barros Neves (1952); Matsuura and Suto (1935)
Austria 24

Austria $\quad 32$

Can. Islands 30

British Isles 30

Germany $\quad 32$

Portugal; cult. mat. 18

Portugal 18-27

Poland 27

unknown 27

Portugal; cult. mat. $\quad 27$

Hungary 27

cult. mat. $\quad 27$

cult. mat. 36

cult. mat. $\quad 45$

cult. mat. 54

7

7

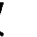

54
36,38

38

38

$32,34,36$

34

34

34

34,36

$34,36,38$

$34,35,36$

7,38

$34,35,36$

18

18

36
36

36

18

34-36

36 
Ornithogalum umbellatum L.

Parnassia palustris L.

Poa nemoralis $L$.

Polygonatum

multiflorum (L.) All.

Polygonatum odoratum (Mill.) Druce

Potentilla anserina L.
St. Jansberg near Mook (Limburg) 
REFERENCES

\begin{tabular}{|c|c|c|}
\hline Author & Origin & $2 n$ \\
\hline $\begin{array}{l}\text { de Barros Neves (1956) } \\
\text { idem }\end{array}$ & $\begin{array}{l}\text { Portugal, France } \\
\text { cult. mat. }\end{array}$ & $\begin{array}{l}54 \\
72\end{array}$ \\
\hline $\begin{array}{l}\text { Banach-Pogan (1956) } \\
\text { Erlandsson (1942) } \\
\text { Hamel (1953) } \\
\text { Matsuura and Suto (1935) } \\
\text { Quezel (1957) } \\
\text { Rozanova (1940); Sokolovskaja and Strelkova } \\
\quad(1960) \\
\text { Erlandsson (1942); Löve and Löve (1944) } \\
\text { Hedberg (1958) } \\
\text { A. Löve (1950); Löve and Löve (1951, 1956) } \\
\text { A. Löve (1950) }\end{array}$ & $\begin{array}{l}\text { Poland } \\
\text { Sweden, Denmark } \\
\text { France } \\
\text { Japan } \\
\text { North Africa } \\
\text { U.S.S.R. } \\
\text { Sweden } \\
\text { Scotland } \\
\text { Iceland } \\
\text { Canada }\end{array}$ & $\begin{array}{l}18 \\
18 \\
18 \\
18 \\
18 \\
\\
18 \\
36 \\
36 \\
36 \\
36\end{array}$ \\
\hline $\begin{array}{l}\text { Avdulov (1928, 1931) } \\
\text { Skalinska (in Skalinska et al., 1961) } \\
\text { Sokolovskaja and Strelkova (1940) } \\
\text { Kiellander (in Löve and Löve, 1942) } \\
\text { Guinochet (1943) } \\
\text { Tateoka (1956) } \\
\text { Armstrong (1937) } \\
\text { Guinochet (1943) } \\
\text { Hooper (in Löve and Löve, 1961) } \\
\text { Jörgensen, Sörensen and Westergaard (1958) } \\
\text { Kiellander (in Löve and Löve 1942) } \\
\text { Löve and Löve (1948, 1956) } \\
\text { Stählin (1929) } \\
\text { Hooper (in Löve and Löve, 1961) }\end{array}$ & $\begin{array}{l}\text { cult. mat. } \\
\text { Poland } \\
\text { Acc. to Löve and Löve, } 1961 \\
\text { Scandinavia } \\
\text { France } \\
\text { cult. mat. } \\
\text { England } \\
\text { France } \\
\text { England, Scotland, The Netherl. } \\
\text { Geenland } \\
\text { Scandinavia } \\
\text { Iceland } \\
\text { unknown } \\
\text { Scotland }\end{array}$ & $\begin{array}{l}28 \\
28 \\
28 \\
28-38,43 \\
33 \\
35 \\
42 \\
42 \\
42 \\
42 \\
42 \\
42 \\
42 \\
56\end{array}$ \\
\hline $\begin{array}{l}\text { van Berg (1933) } \\
\text { Dark (in Maude, 1939) } \\
\text { Junell (in Löve and Löve, 1942) } \\
\text { Polya (1950) } \\
\text { Suomalainen (1947) } \\
\text { Therman (1953) } \\
\text { Suomalainen (1947) } \\
\text { Therman-Suomalainen (1949) } \\
\text { Dark (in Maude, 1939) }\end{array}$ & $\begin{array}{l}\text { cult. mat. } \\
\text { England } \\
\text { Scandinavia } \\
\text { Hungary } \\
\text { Sweden, Finland } \\
\text { acc. to Löve and Löve, } 1961 \\
\text { cult. mat. } \\
\text { acc. to Löve and Löve, } 1961 \\
\text { England }\end{array}$ & $\begin{array}{l}18 \\
18 \\
18 \\
18 \\
18 \\
18 \\
20 \\
20 \\
30\end{array}$ \\
\hline $\begin{array}{l}\text { van Berg (1933) } \\
\text { Fernandes (1950) } \\
\text { Junell (in Löve and Löve, 1942) } \\
\text { Hasegawa (1933) } \\
\text { Mori (1957) } \\
\text { Suomalainen (1947) } \\
\text { Therman (1953) } \\
\text { Therman-Suomalainen (1949) } \\
\text { Maude (1939) }\end{array}$ & $\begin{array}{l}\text { cult. mat. } \\
\text { Portugal } \\
\text { Scandinavia } \\
\text { unknown } \\
\text { Italy } \\
\text { Sweden, Finland, Denmark } \\
\text { acc. to Löve and Löve, 1961 } \\
\text { idem } \\
\text { England }\end{array}$ & $\begin{array}{l}20 \\
20 \\
20 \\
20 \\
20 \\
20 \\
20 \\
26,28,30 \\
26,28,30\end{array}$ \\
\hline $\begin{array}{l}\text { Löve and Löve (1942) } \\
\text { Löve and Löve (1956) } \\
\text { Polya (1949) } \\
\text { Popoff (1935) } \\
\text { Skalinska and Czapick (1959) } \\
\text { Turesson (1938) } \\
\text { Erlandsson (1942) }\end{array}$ & $\begin{array}{l}\text { Sweden } \\
\text { Iceland } \\
\text { Hungary } \\
\text { cult. mat. } \\
\text { Poland } \\
\text { North America, Sweden } \\
\text { Sweden, Denmark }\end{array}$ & $\begin{array}{l}28 \\
28 \\
28 \\
28 \\
28 \\
28 \\
42\end{array}$ \\
\hline
\end{tabular}


TABle II (Cont.)

Species

Potentilla anserina $\mathrm{L}$.

Prunella vulgaris L.

802
$2 n$

Origin

Coll. no.

Ranunculus acris $\mathbf{L}$.

Molenpolder near Westbroek (Utrecht) idem

Between Houten and Schalkwijk (Utrecht) Fort Hoofddijk near Utrecht (Utrecht) near Maarn (Utrecht) near Oosterend, Terschelling (Friesland)
14

14

14

14

14
Ranunculus auricomus L.

Ranunculus repens $L$. near Gorkum (S. Holland) 32

Lohmanskamp near Ootmarsum (Overijss.) 32

Between Boxtel and Best (N. Brabant) 32

Camerig near Epen (Limb.) 32

Eyserbos, Eys (Limburg) 32

Rhijnauwen near Bunnik (Utrecht) 32

near Epen (Limburg) 32

Between Terziet and Epen (Limburg) $\quad 32$

near Mechelen (Limburg) 32

Biesbos (N. Brabant) 32

near Hardenberg (Overijssel) 32

Between Gulpen and Slenaken (Limburg) 32

Between Houten and Schalkwijk (Utrecht) 32

St. Jansberg near Mook (Limburg) 32

Fort Hoofddijk near Utrecht (Utrecht) 32

near Maarn (Utrecht) $\quad 32$

Mereveld near Utrecht (Utrecht) 32

Schone Grub near Rijckholt (Limburg) 32

Schweiberger Bos near Mechelen (Limburg) 32

Grebbeberg, Rhenen (Utrecht) 32

Quackjeswater near Rockanje (S. Holland) 32

Betw. Ermelo and Nw. Millingen (Gelderl.) 32

Betw. Ermelo and Nw. Millingen (Gelderl.) 26 
REFERENCES

\begin{tabular}{|c|c|c|}
\hline Author & Origin & $2 n$ \\
\hline Roscoe (1927) & North America & 42 \\
\hline Böcher (1949) & \multirow{2}{*}{$\begin{array}{l}\text { Faroes, Denmark, Sweden, U.S.S.R. } \\
\text { Austria, France, Portugal, Canada } \\
\text { unknown } \\
\text { Iceland } \\
\text { Norway, Denmark, Canada, Faroes } \\
\text { Japan } \\
\text { Central Europe } \\
\text { Austria }\end{array}$} & 28 \\
\hline $\begin{array}{l}\text { Levitsky (1940) } \\
\text { Löve and Löve (1956) } \\
\text { Böcher (1940) } \\
\text { Hara (1954) } \\
\text { Hruby (1932) } \\
\text { Mattick (in Tischler, 1950) }\end{array}$ & & $\begin{array}{l}28 \\
28 \\
32 \\
32 \\
32 \\
32\end{array}$ \\
\hline $\begin{array}{l}\text { Sorokin (1924, 1927, a-b-c-d-) } \\
\text { Groonen (1939) } \\
\text { Hara and Kurosawa (1956) } \\
\text { Hess (1953) } \\
\text { Kurita (1956, 1957, 1958) } \\
\text { Langlet (1932) } \\
\text { Larter (1932); Miyaji (1927) } \\
\text { Löve and Löve (1944) } \\
\text { Löve and Löve (1956) } \\
\text { Marsden Jones and Turrill (1935) } \\
\text { Matsuura and Suto (1935) } \\
\text { Mulligan (1959) } \\
\text { Polya (1950) } \\
\text { Tomaszewski (1959) } \\
\text { Hara and Kurosawa (1956) } \\
\text { Langlet (1932) } \\
\text { Senjaninova (1926) } \\
\text { Nygren (in Löve and Löve, 1948) }\end{array}$ & $\begin{array}{l}\text { cult. mat. } \\
\text { cult. mat. } \\
\text { Japan } \\
\text { Switzerland } \\
\text { acc. to Löve and Löve (1961) } \\
\text { Sweden } \\
\text { unknown } \\
\text { Sweden } \\
\text { Iceland } \\
\text { England } \\
\text { Japan } \\
\text { Canada } \\
\text { Hungary } \\
\text { Poland } \\
\text { Japan } \\
\text { Germany } \\
\text { U.S.S.R. } \\
\text { Scandinavia }\end{array}$ & $\begin{array}{l}12-18 \\
14 \\
14 \\
14 \\
14 \\
14 \\
14 \\
14 \\
14 \\
14 \\
14 \\
14 \\
14 \\
14 \\
28 \\
28 \\
28,29-32 \\
28,56\end{array}$ \\
\hline $\begin{array}{l}\text { Häfliger (1943); Rutishauser (1954) } \\
\text { Kleczkowska (in Skalinska et al., 1961) } \\
\text { Langlet (1932); Larter (1932) } \\
\text { Levitsky (1940) } \\
\text { Löve and Löve (1956) } \\
\text { Mattick (in Tischler, 1950) } \\
\text { de Barros Neves (1944) } \\
\text { Polya (1950) } \\
\text { Rousi (1956) } \\
\text { Häfliger (1943) } \\
\text { Rousi (1956) }\end{array}$ & $\begin{array}{l}\text { Switzerland } \\
\text { Poland } \\
\text { cult. mat. } \\
\text { acc. to Löve and Löve, } 1961 \\
\text { Iceland } \\
\text { Austria } \\
\text { Portugal } \\
\text { Hungary } \\
\text { Finland } \\
\text { Switzerland } \\
\text { Finland }\end{array}$ & $\begin{array}{l}32 \\
32 \\
32 \\
32 \\
32 \\
32 \\
32 \\
32 \\
32 \\
40,48 \\
40,48\end{array}$ \\
\hline $\begin{array}{l}\text { Matsuura and Suto (1935) } \\
\text { Mattick (in Tischler, 1950) } \\
\text { Hocquette (1922); Langlet (1927); Larter (1932) } \\
\text { Kurita (1958) } \\
\text { Löve and Löve (1956) } \\
\text { Matsuura and Suto (1935) } \\
\text { Mattick (in Tischler, 1950) } \\
\text { de Barros Neves (1950) } \\
\text { Polya (1949) } \\
\text { Sokolovskaja (1960) } \\
\text { Sokolovskaja and Strelkova (1950) }\end{array}$ & $\begin{array}{l}\text { Japan } \\
\text { Austria } \\
\text { unknown } \\
\text { acc. to Löve and Löve, } 1961 \\
\text { Iceland } \\
\text { cult. mat. } \\
\text { Austria } \\
\text { Portugal } \\
\text { Hungary } \\
\text { Sachalin } \\
\text { U.S.S.R. }\end{array}$ & $\begin{array}{l}16 \\
16 \\
32 \\
32 \\
32 \\
32 \\
32 \\
32 \\
32 \\
32 \\
32\end{array}$ \\
\hline $\begin{array}{l}\text { Scheerer (1940) } \\
\text { Löve and Löve (1942) }\end{array}$ & $\begin{array}{l}\text { Germany } \\
\text { Sweden }\end{array}$ & $\begin{array}{l}26 \\
42\end{array}$ \\
\hline
\end{tabular}


Table II (Cont.)

\begin{tabular}{clll}
\hline Species & Coll. no. & Origin & 2n \\
\hline
\end{tabular}

Rorippa amphibia (L.) Besser $322 \quad$ Rhijnauwen near Bunnik (Utrecht)

Symphytum officinale L.

Rhijnauwen near Bunnik (Utrecht)

Betw. Jutphaas and IJsselstein (Utrecht)

Fort Hoofddijk near Utrecht (Utrecht)

Teucrium scorodonia L.

Schweiberger Bos near Mechelen (Limb.)

Trifolium dubium Sibth.

K 98 Walcheren near Middelburg (Zeeland)

Trifolium ornithopodioides L. K $91 \quad$ Isle of Walcheren, (Zeeland)

Trifolium scabrum L.

K 92 West-Kapelle, Walcheren (Zeeland)

$\mathrm{K} 121$ idem

- idem $(14 \times)$

- $\quad$ Renesse (Schouwen), Zeeland

Trifolium subterraneum $\mathbf{L}$.

K 96 West-Kapelle, Walcheren (Zeeland)

Urtica dioica L.

Urtica urens L.
St. Jansberg near Mook (Limburg) Rhijnauwen near Bunnik (Utrecht) Maarn (Utrecht)

Schone Grub near Rijckholt (Limburg) Grebbeberg near Rhenen (Utrecht) near Utrecht (Utrecht)

in a garden, Utrecht (Utrecht)
Valeriana officinalis L. (s.1.) $\quad 626$
Schweiberger Bos near Mechelen (Limb.)

Geerendal, Schin op Geul (Limburg)

Between Baarn and Eemdijk (Utrecht)

Quackjeswater, Rockanje (S. Holland) near IJsselstein (Utrecht) 


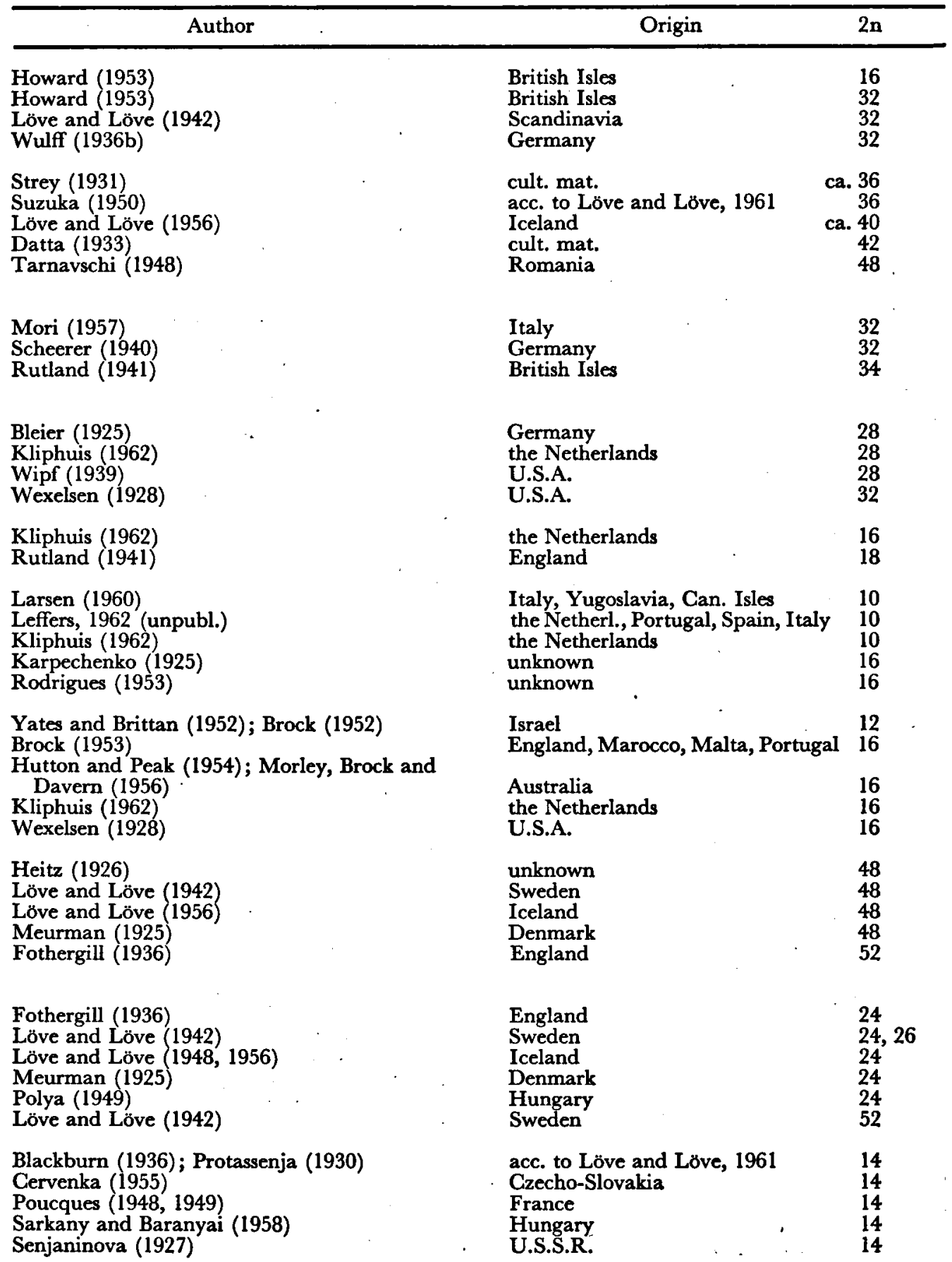


Valeriana officinalis L. (s.1.)

Veronica officinalis $\mathbf{L}$.

Vicia cracca L.

Viola riviniana $R$ chb.

21 different localities in the Netherlands 
REFERENCES

\begin{tabular}{|c|c|c|}
\hline Author & Origin & $2 n$ \\
\hline $\begin{array}{l}\text { Skalinska (1950) } \\
\text { Walther (1949) } \\
\text { Blackburn (1936); Protassenja (1930) } \\
\text { Polya (1949); Sarkany and Baranyai (1958) } \\
\text { Senjaninova (1927) } \\
\text { Skalinska (1945, 1947) } \\
\text { Skalinska (1950) } \\
\text { Walther (1949) } \\
\text { Cervenka (1955) } \\
\text { Hegnauer and Meyers (1958) } \\
\text { Löve and Löve (1956) } \\
\text { Meurman (1925) } \\
\text { Sarkany and Baranyai (1958) } \\
\text { Skalinska (1945, 1947); Todd (1942) } \\
\text { Skalinska (in Skalinska et al., 1959) }\end{array}$ & $\begin{array}{l}\text { Poland } \\
\text { Germany } \\
\text { acc. to Löve and Löve, } 1961 \\
\text { Hungary } \\
\text { U.S.S.R. } \\
\text { England } \\
\text { Poland } \\
\text { Germany } \\
\text { Czecho Slovakia } \\
\text { the Netherlands } \\
\text { Iceland } \\
\text { Denmark } \\
\text { Hungary } \\
\text { England } \\
\text { Poland }\end{array}$ & $\begin{array}{l}14 \\
14 \\
28 \\
28 \\
28 \\
28 \\
28 \\
28 \\
56 \\
56 \\
56 \\
56 \\
56 \\
56 \\
56\end{array}$ \\
\hline $\begin{array}{l}\text { Böcher (1944) } \\
\text { Huber (1927) } \\
\text { Afanasiyeva and Meshkova (1961) } \\
\text { Simonet (1934) } \\
\text { Böcher (1944) } \\
\text { Löve and Löve (1956) }\end{array}$ & $\begin{array}{l}\text { Sweden } \\
\text { cult. mat. } \\
\text { unknown } \\
\text { France } \\
\text { Faroes, Iceland, Denmark, Norway, } \\
\text { Germany, Finland, Sweden } \\
\text { Iceland }\end{array}$ & $\begin{array}{l}18 \\
32-37 \\
34 \\
34,36 \\
36 \\
36\end{array}$ \\
\hline $\begin{array}{l}\text { Renkonen and Therman (1952); Rousi (1961) } \\
\text { Sakamura (1914) } \\
\text { Sveshnikova (1927) } \\
\text { Baksay (1954) } \\
\text { Rousi (1961) } \\
\text { Ryka (1954) } \\
\text { Senn (1938); Moriya and Kondo (1950) } \\
\text { Renkonen and Therman (1952) } \\
\text { Rousi (1961) } \\
\text { Jörgensen, et al. (1958) } \\
\text { Ledingham (1957) } \\
\text { Löve and Löve (1944) } \\
\text { Löve and Löve (1956) } \\
\text { Rousi (1961) }\end{array}$ & $\begin{array}{l}\text { cult. mat. } \\
\text { Japan } \\
\text { U.S.S.R. } \\
\text { Hungary } \\
\text { France, Austria, Hungary, U.S.S.R. } \\
\text { Poland } \\
\text { unknown } \\
\text { cult. mat. } \\
\text { cult. mat. } \\
\text { Greenland } \\
\text { Canada } \\
\text { Sweden } \\
\text { Iceland } \\
\text { Finland, Sweden, Denmark, } \\
\text { England, the Netherlands, } \\
\text { Germany, Poland, France, } \\
\text { Austria, Switzerland, U.S.S.R., } \\
\text { Canada, New Zeal. } \\
\text { Poland } \\
\text { US S.R. Germany }\end{array}$ & $\begin{array}{l}12 \\
12 \\
12 \\
14 \\
14 \\
14 \\
14 \\
27,28 \\
27 \\
28 \\
28 \\
28 \\
28\end{array}$ \\
\hline $\begin{array}{l}\text { Clausen (1927); Gershoy (1934) } \\
\text { Fothergill (1944); Valentine (1941, 1949, 1950) } \\
\text { Gadella (1963, in press) } \\
\text { Schmidt (1961); Schöfer (1954) } \\
\text { Gadella (1963, in press) }\end{array}$ & $\begin{array}{l}\text { Denmark } \\
\text { England } \\
\text { Germany, Belgium } \\
\text { Germany } \\
\text { the Netherlands }\end{array}$ & $\begin{array}{l}40 \\
40 \\
40 \\
40 \\
35,40,45 \\
46,47\end{array}$ \\
\hline Valentine (1949) & England & \\
\hline
\end{tabular}


TABLE II (Cont.)

\begin{tabular}{clll}
\hline Species & Coll. no. & Origin & 2n \\
\hline Viola palustris L. & 248 & Betw. Ermelo and Nw. Millingen (Gelderl.) & 48 \\
& 83 & near Maarn (Utrecht) & 48
\end{tabular}

TABLE III

Species with chromosome numbers not determined before.

\begin{tabular}{c|l|l|l}
\hline coll. no. & \multicolumn{1}{|c|}{ species } & \multicolumn{1}{|c|}{ origin } & 2n \\
\hline 667 & Lysimachia thyrsiflora L. & $\begin{array}{l}\text { between Baarn and } \\
\text { Eemdijk, Utrecht. }\end{array}$ & \\
720 & Scutellaria galericulata L. & $\begin{array}{l}\text { near IJsselstein, Utrecht. } \\
\text { near IJsselstein, Utrecht. }\end{array}$ & 30 \\
528 & Scrophularia neesii Wirtg. & near Epen, Terziet, Limburg & 78 \\
613 & Scrophularia balbisii Hornem. & near Epen,
\end{tabular}

Discussion

A. Species with chromosome numbers in accordance with the literature:

1. Thymus pulegioides L.

Previously Thymus pulegioides $\mathrm{L}$. and Thymus serpyllum L. (s. str.) were considered as subspecies of Thymus serpyllum L. (s.l.), named: Thymus serpyllum L. ssp. chamaedrys (Fr.) Vollm. and Th. serpyllum L. ssp. angustifolium (Pers.) Vollm. respectively.

The first is a plant of calcareous soil, the latter of diluvial sand. The subspecies have different chromosome numbers, ssp. angustifolium: $2 \mathrm{n}=24$, and ssp. chamaedrys: $2 \mathrm{n}=28$. Besides morphological and ecological differences, cytological evidences also supports the splitting into two species.

\section{Armeria maritima (Mill.) Willd.}

Besides plants from the Dutch coast, also plants from Belgium were investigated. The origin of the Belgian plants was a typical VioletumCalaminariae on rubble of a zinc mine, near La Calamine. The chromosome number $2 n=18$ could be determined for all nine investigated plants.

B. Species with intraspecific cytological variation.

This group can be divided as follows:

1. Species with an intraspecific aneuploid series.

2. Species with an intraspecific euploid series.

3. Species with both intraspecific euploidy and aneuploidy. 
REFERENCES

\begin{tabular}{llr}
\hline \multicolumn{1}{c}{ Author } & & Origin \\
\hline Bold and Gershoy (1934) & Canada & 48 \\
Clausen (1931) & Denmark & 48 \\
Fernandes (1950) & Portugal & 48 \\
Gadella (1963, in press) & the Netherlands, Belgium & 48 \\
Gershoy (1928) & North America & 48 \\
Jörgensen, et al., (1958) & Greenland & 48 \\
Löve and Löve (1956) & Iceland & 48 \\
\hline
\end{tabular}

The distinguished groups will be described successively.

\section{Species with an intraspecific aneuploid series.}

To this group belong the following investigated species: Aethusa cynapium L.; Alisma plantago-aquatica L.; Artemisia vulgaris L.; Baldellia ranunculoides (L.) Parl.; Epipactis helleborine (L.) Crantz.; Filipendula ulmaria (L.) Maxim.; Hippuris vulgaris L.; Liparis loeselii (L.) Rich.; Listera cordata (L.) R.Br.; Listera ovata (L.) R.Br.; Lysimachia nemorum L.; Lysimachia nummularia L.; Mercurialis perennis L.; Myosotis sylvatica Ehr. ex Hoffm.; Origanum vulgare L.; Rhynchospora alba (L.) Vahl.; Teucrium scorodonia L.; Trifolium dubium Sibth.; Trifolium ornithopodioides L.; Trifolium scabrum L.; Trifolium subterraneum L.; Urtica dioica L.; Viola riviniana $\mathrm{Rchb}$.

From the above mentioned species the following will be discussed further:

\section{Artemisia vulgaris $\mathrm{L}$.}

A number of species of the genus Artemisia have the basic number $\mathrm{x}=8$ and $\mathrm{x}=9$. Artemisia vulgaris belongs either to both groups or the material from India may belong to another species.

\section{Filipendula ulmaria (L.) Maxim.}

In the same roottip metaphaseplates with $2 n=14$ and $2 n=16$ were found. These chromosome numbers were known before. But they were always referred to separate plants and are not known to occur within the same individual.

The chromosome number $2 \mathrm{n}=15$, mentioned in the literature might possibly indicate an hybridisation between plants with $2 n=14$, and plants with $2 \mathrm{n}=16$.

3. Listera ovata (L.) R. Br.

The chromosome numbers $2 \mathrm{n}=34,35,36$ and 37 were determined. The numbers 35,36 and 37 are caused by respectively one, two or three small additional chromosomes, not present in the 34 individuals.

Morphological differences between plants with different chromosome numbers are not present. Apparently the extra chromosomes have to be considered as supernumeraries as suggested by MacMahon 
in his study of the behaviour of these additional chromosomes during meiosis. (1936).

\section{Lysimachia nummularia $L$.}

For this species the chromosome number $2 \mathrm{n}=36$ is mentioned in the literature; meanwhile Lysimachia nemorum $L$. has the number $2 \mathrm{n}=18$.

This might suggest a basic number $\mathrm{x}=9$, at least for a part of this genus.

The determined chromosome number in our investigations, appeared to be $2 n=32$ and $2 n=16$, indicating a basic number $x=8$.

In plants of Lysimacha nummularia from two different localities the chromosome number $2 n=43$ was counted.

\section{Mercurialis perennis $L$.}

In Hungary BAKsay (1957) found the chromosome number $2 n=42$ in two plants of this species. According to this author, the material investigated by MEURMAN (1925) having the chromosome number $2 \mathrm{n}=64$, belongs to Mercurialis longistipes (Borbas) Baks., which also occurs in Hungary. Baksay examined eight plants and found $2 \mathrm{n}=64$.

In the Netherlands Mercurialis perennis L. appeared to have $2 \mathrm{n}=64$ in female plants and $2 \mathrm{n}=66$ in male plants.

Further research concerning the constancy of the difference between the chromosome numbers of the male- and the female plants in different populations in the Netherlands is planned.

The results of our investigation concerning the female plants having the chromosome number $2 \mathrm{n}=64$ might indicate that the opinion of Baksay with regards to the investigations of Meurman is incorrect.

\section{Trifolium scabrum $L$.}

This species is rare in the Netherlands. Plants from the province of Zeeland (Walcheren and Schouwen), were collected and investigated. They all had the diploid number $2 \mathrm{n}=10$ (KLIPHuIs 1962, LeFFers, unpubl.). Leffers also counted $2 \mathrm{n}=10$ in material from Portugal, Spain and Italy.

\section{Trifolium subterraneum $L$.}

The chromosome number in this species is $2 \mathrm{n}=16$.

In plants from Israël Brock (1953) found $2 \mathrm{n}=12$. This author suggested that the difference in chromosome number might be the result of a rearrangement of the chromosomes without loss of material.

\section{Viola riviniana $\mathrm{R}$ chb.}

Extensive experimental research on this species growing in the Netherlands has been done by Gadella (1963). The material investigated so far, seems to be more like that from Britain than that from southern Germany, because in different populations extra chromosomes appeared to be present without any correlation with morphological characters. Morphological the species Viola riviniana 
Rchb. is closely related to the diploid species Viola reichenbachiana Jord. ex Bor., but several diagnostic characters permit an easy distinction between the two species at least in the Netherlands.

In 1962, in the south of the province of Limburg, some obviously intermediate plants were collected. These plants are subjected to further investigations in Utrecht.

\section{Species with an intraspecific euploid series.}

The following investigated species belong to this group:

Actaea spicata L.; Alliaria petiolata (Bieb.) Cavara et Grande; Butomus umbellatus L.; Campanula rotundifolia L.; Cardamine amara L.; Chrysanthemum leucanthemum L.; Comarum palustre L.; Euphorbia cyparissias L.; Galium hercynicum Weig.; Galium palustre L.; Galium uliginosum L.; Glechoma hederacea L.; Halimione portulacoides (L.) Aellen.; Hieracium pilosella L.; Lamium galeobdolon (L.) L.; Lonicera periclymenum L.; Lysimachia vulgaris L.; Ornithogalum umbellatum L.; Parnassia palustris L.; Potentilla anserina L.; Prunella vulgaris L.; Ranunculus auricomus L.; Ranunculus repens L.; Rorippa amphibia (L.) Bessu.; Valeriana officinalis L.; Viola palustris $\mathrm{L}$.

Next species will be discussed further:

\section{Butomus umbellatus $L$.}

According to A. Löve (1951), in Sweden the triploid $(2 \mathrm{n}=39)$ races can be distinguished from the diploid ones $(2 n=26)$ morphologically, owing to their sterility and high degree of bulbifery. Whether this is also correct for other parts of the area, in particular in the Netherlands, can only be proved by further investigations.

\section{Campanula rotundifolia L. (s.l.)}

A survey of cytological data concerning this species up to the present was given by GADELLA (1962).

Crossings between diploids, tetraploids and hexaploids are the subject of further research by this author.

\section{Chrysanthemum leucanthemum L. (s.l.)}

According to Böcher and LARSEN (1957) the diploid races of this complex species belong to Chrysanthemum leucanthemum L. (s. str.) and the tetraploid ones to Chrysanthemum ircutianum Turcz. (s.1.), although some diploid varieties show some morphological resemblance to the tetraploids. The material examined by us proved to be tetraploid. Diploid plants have not been found in the Netherlands yet.

\section{Euphorbia cyparissias L.}

Tetraploids $(2 n=40)$ and sterile diploids $(2 n=20)$ are found in Canada. Tetraploids are also common in England, the sterile diploids escaped there from gardens occasionally. On the continent of Europe tetraploids should be more numerous than diploids. However, Pritchard (1959), found a fertile diploid plant in a wood near Fontainebleau. 
MOORE and Lindsay (1953) gave a diameter of 22-26 micron for the sterile and empty pollen of the diploids and one of 35-40 micron for the fertile pollen of the tetraploids. The plants investigated by us were diploid. The pollen appeared to be normal, with a diameter of 32-37 micron so that these may be regarded as fertile.

\section{Galium palustre L.}

Only plants with $2 n=24$ were found in the Netherlands. (KLIPHuIs, 1962). The chromosome numbers $2 n=48$ and $2 n=96$ are known from other countries. According to CLAPHAM (1949) the diploids and octoploids can be separated morphologically. The tetraploids, however, are intermediate. For that reason this author suggested that the three forms should be treated as cytological subspecies rather than separate species.

\section{Glechoma hederacea L.}

In Hegi's "Flora von Mitteleuropa" (1906/1931), the species Glechoma hederacea L. is divided into two subspecies: ssp. glabriusculum (Neilr.) Gams, ( = Glechoma hederacea L. s. str.) and ssp. hirsutum (W. et K.) Hermann, (= Glechoma hirsuta W. et K.). According to D. Löve and J. P. BERnARD (1959) the diploid Glechoma hederacea L. s. str., with $2 n=18$ is unknown in Canada, only the tetraploid large flowering and pubescent form is found there.

In the Netherlands the chromosome number $2 n=36$ could be determined in four cases.

Table four gives a survey of the differential characters of the two subspecies according to Hegi and the characters of the plants investigated cytologically by us.

The Dutch material appears to have many characters of the subspecies glabriusculum, except that it is not diploid but tetraploid.

An extensive cytotaxonomic study may give more clarity about the taxonomic significance of the diploid and tetraploid races.

\section{Hieracium pilosella L.}

In Sweden this species was investigated extensively by Turesson and Turesson (1960). Plants with $2 n=36$ appeared to be amphimictic, the plants with $2 n=45$, or more chromosomes, amphiapomictic.

All plants investigated by us had the chromosome number $2 \mathrm{n}=36$; according to the results of Turesson and Turesson these plants are possibly amphimictic too.

8. Lamium galeobdolon $\mathrm{L}$.

According to TuResson (1938) this species is characterized by: "a diploid type being distributed in the north and a taller, late flowering, more robust, tetraploid southern type. The tetraploid is sometimes treated as a variety, or even as a separate species: Galeobdolon montanum Rchb."

The material investigated by us appeared to be tetraploid. 
TABLE IV

A comparison of the morphological and cytological characters of the two subspecies of Glechoma hederacea L.

\begin{tabular}{|c|c|c|c|c|}
\hline \multirow{2}{*}{. . } & \multicolumn{4}{|c|}{ population number } \\
\hline & 353 & 550 & 664 & 714 \\
\hline $\begin{array}{l}\text { subsp. glabriusculum (Neilr.) Gams. } \\
\text { 1a. stem and leaves with scattered patent-retrorse hairs. } \\
\text { 2a. petioles shorter than internodes } \\
\text { 3a. width of leafs up to } 3 \mathrm{~cm} \text {. } \\
\text { 4a. corolla violet-blue, up to } 3 \mathrm{~cm} \text {. long. } \\
\text { 5a. posterior stamens of the corolla much shorter than the } \\
\text { upper lip of the corolla. } \\
\text { 6a. calyx teeth triangular, } 1 / \mathrm{s} \times \text { calyx tube, aristated. } \\
\text { 7a. throughout the area of the species. } \\
\text { 8a. chromos. number: } 2 \mathrm{n}=18 \text { (D. Löve, 1959) }\end{array}$ & $\begin{array}{l}\mathbf{x} \\
\mathbf{x} \\
\mathbf{x} \\
\mathbf{x}\end{array}$ & $\begin{array}{l}\mathbf{x} \\
\mathbf{x} \\
\mathbf{x} \\
\mathbf{x}\end{array}$ & $\begin{array}{l}\mathbf{x} \\
\mathbf{x} \\
\mathbf{x} \\
\mathbf{x}\end{array}$ & $\begin{array}{l}\mathbf{x} \\
\mathbf{x} \\
\mathbf{x} \\
-\end{array}$ \\
\hline $\begin{array}{l}\text { subsp. hirsutum (W. et K.) Herm. } \\
\text { 1b. stem indumentum pilose to appressed scabrous-hirsute. } \\
\text { 2b. petiole equalling the internode. } \\
\text { 3b. width of leafs } 3-4 \mathrm{~cm} \text {. long (up to } 7 \mathrm{~cm} \text {.) } \\
\text { 4b. corolla bright blue, up to } 3 \mathrm{~cm} \text {. long. } \\
\text { 5b. posterior stamens attaining sinus of upper lip. } \\
\text { 6b. calyx teeth narrowly lanceolate with a long slender, } \\
\text { often violet arista. } \\
\text { 7b. S. and S.E. Europe. } \\
\text { 8b. chromos. number: } 2 \mathrm{n}=36 \text { (D. Love, 1959) }\end{array}$ & $\mathbf{x}$ & $\mathbf{x}$ & $\mathbf{x}$ & $\mathbf{x}$ \\
\hline
\end{tabular}

\section{Lonicera periclymenum L.}

Up to the present only a few hexaploid Lonicera species are known, all from southeast Asia. (Janaki-Ammal and Saunders, 1939). The European plants, as far as investigated, are diploid or tetraploid. All the material examined by the present authors turned out to be hexaploid.

\section{Parnassia palustris $\mathrm{L}$.}

According to A. Löve (1950) the tetraploid plants of this species, with the chromosome number $2 n=36$, have a circumpolar distribution, the diploids, with $2 \mathrm{n}=18$ an eurasiatic. This is confirmed by Rozanova (1940, 1946) and Banach-Pogan (1956).

The diploids and tetraploids can be separated morphologically. In contradiction to what could have been expected, tetraploid plants of Parnassia palustris L. were found in the Netherlands only.

For a final conclusion it is desirable to undertake a more detailed study of material present in the Netherlands to ascertain wether Parnassia palustris L. is mainly occuring as a tetraploid or as a diploid.

\section{Valeriana officinalis L. (s.l.)}

Sometimes this collective species is split into four species: Valeriana officinalis L. (s. str.), with $2 \mathrm{n}=14$; Valeriana collina Wallr., with $2 \mathrm{n}=28$; Valeriana procurrens Wallr., with $2 \mathrm{n}=56$ and Valeriana sambucifolia Mikan, with $2 \mathrm{n}=56$. 
These four species can be distinguished very well morphologically, but in some parts of the area the situation is troubled by hybridisation.

In the Netherlands Hegmann and Meyers (1958), found octoploids only. This could be confirmed by our investigations. Hegmann and Meyers do not underscribe the splitting as mentioned above. WALTHER (1949), gives a distribution map of the European species. According to her the octoploid Valeriana procurrens Wallr. $(2 n=56)$ occurs in the Netherlands only.

\section{Species with both intraspecific euploidy and aneuploidy.}

Galium aparine L.; Iris pseudacorus L.; Poa nemoralis L.; Polygonatum multiflorum (L.) All.; Polygonatum odoratum (Mill.) Druce; Ranunculus acris L.; Symphytum officinale L.; Urtica urens L.; Veronica officinalis L.; Vicia cracca L.

Next species will be discussed further:

\section{Galium aparine L.}

Plants with the chromosome number $2 \mathrm{n}=64$ seem to be most common, but this species occurs also as a di-, tetra-, hexa- and octoploid, with $2 \mathrm{n}=22,44,66$ and 88 respectively.

In the metaphaseplates of cells of $2 n=64$, two large chromosomes are present. Böcher et al. (1955), suggested that each of these two large chromosomes may have arisen by fusion of two short chromosomes.

\section{Symphytum officinale L.}

Three cytotypes of this species, with $2 n=26,40$ and 48 respectively, were found in a limited area. Morphological and ecological differences are not known.

Investigations to this extend will be undertaken in the future. In addition the possibility of crossings between the cytotypes will be the subject of research.

G. Species with chromosome numbers not determined before.

1. Lysimachia thyrsiflora L.

DAHLGRen (1916) did not succeed to establish the exact chromosome number of this species $(2 n=c a .40)$.

The chromosome number of the plants investigated by us could be determined without doubt as $2 \mathrm{n}=54$.

\section{Scutellaria galericulata L.}

Up to now this species was investigated only once. However, the chromosome number could not be determined exactly ( $2 n=c a .32)$.

In the somatic metaphaseplates of roottip cells of plants studied by us the number $2 n=30$ was counted repeatedly. 


\section{Scrophularia neesii Wirtg. and Scrophularia balbisii} Hornem.

The first species mentioned has a chromosome number of $2 n=52$, the second $2 \mathrm{n}=78$.

Both species are also considered as two subspecies of Scrophularia alata $\mathrm{L}$. The cytological data, however, indicate that most likely these taxa are separate species. Further research in order to ascertain if there is a genetic barriere between the two species present, is planned.

\section{ACKNOWLEDGEMENT}

This investigation was carried out at the Botanical Museum and Herbarium, Utrecht, director Prof. Dr. J. Lanjouw. Partly it was made possible by a grant of the Netherlands Organisation of Pure Scientific Research.

The authors are much indebted to miss J. Niers for her technical assistance.

\section{REFERENCES}

Afanastyeva, N. G. and L. Z. Meshrova, 1961. Bot. Zhurn. 46: 247-259. Armstrong, J. M. 1937. Canad. Jour. Res. C. 15: 281-297.

Avduzov, N. P. 1928. Drievnik vsesojuznogo Sezda Botanikov Leningrade: 65-66. -. 1931. Bull. Appl. Bot. Genet. \& Plant Breeding Suppl. 43: 1-438. Baez-Mayor, A. B. 1934. Cavanillesia 6: 59-103.

BAKSAY, L. 1954. Ann. Hist. Nat. Mus. Natl. Hung. S.N. 5: 139-148.

-1956. Ann. Hist. Nat. Mus. Natl. Hung. S.N. 7: 321-334.

_-. 1957b. Ann. Hist. Nat. Mus. Natl. Hung. S.N. 9: 169-174. 1959. IX Int. Bot. Congr. vol. II, abstracts: 16.

Bamford, R. and A. Gershoy, 1930. Univ. Vermont Agric. Bull. 325: 1-56. Banach-Pogan, E. 1955. Acta Soc. Bot. Polon. 24: 275-286. 1956. Acta Soc. Bot. Polon. 25: 675-679.

BARBER, H. N. 1942. Journ. Genet. 43: 97-103.

BJöRKQUisT, I. 1959. Bot. Not. 112: 377-380.

BleIER, H. 1925. Jahrb. Wiss. Bot. 64: 604-636.

Böcher, T. W. 1936. Hereditas 22: 269-277.

ㄷ. 1938a. Sv. Bot. Tidskr. 32: 346-361.

- 1940. Dansk. Bot. Ark. 10, 3: 1-15.

․ 1944. Dansk. Bot. Ark. 11, 7: 1-120.

- 1 1949. New Phytol. 48: 285-314.

- 1960. Dansk Vid. Selsk. Biol. Skr. 11, 4: 1-69.

Böcher, T. W. and K. Larsen. 1950. Medd. Gronl. 147, 6: 1-32.

and 1957. Watsonia 4: 11-16.

Böcher, T. W., K. Laksen and K. RaHN, 1955. Dansk Vid. Selsk. Biol. Skr. 8, 3: $1-31$.

Bord, H. G. and A. Gershoy. 1934. Vermont Agric. Exp. Sta. Bull. 378: 1-36.

BRITron, D. M. 1951. Brittonia 7: 233-266.

Brock, R. D. 1953. Nature 171: 939.

CAstro, D. DE and F. C. Fontes. 1946. Broteria 15: 38-46.

Cand M. Noronha-WAgNeR, 1950. Genetica Iberica 3: 75-82.

Cervenka, V. B. 1955. Preslia 27: 234-242.

Christofr, M. and A. Poporf. 1933. Planta 20: 440-447.

Clapham, A. R. 1949. In Wilmott, A. J. (ed.): British Flowering Plants and Modern Systematic Methods, 1949: 72-74.

Clausen, J. 1931. Bot. Tidsskr. 41: 317-335. and 1940 . Carnegie Inst. Publ. 520: 1-452. 
Coonen, L. P. 1939. Am. Journ. Bot. 26: 48-58.

Cooper, D. C. and K. L. Mahony. 1935. Am. Journ. Bot. 22: 843-848.

Dahlgren, K. V. O. 1916. Sv. Vet. Akad. Handl. 56, 4: 1-80.

DATTA, S. 1933. Journ. Ind. Bot. Soc. 12: 131-152.

Dowrick, G. J. 1952. Heredity 6: 365-375.

Duckert, M. M. and C. Favarger. 1956. Ber. Schweiz. Bot. Ges. 66: 134-146. Ehrenberg, L. 1945. Bot. Not. 1945: 430-437.

EHRENDORFER, F. 1949. Öster. Bot. Zeitschr. 96: 109-138.

- 1953. Osterr. Bot. Zeitschr. 100: 616-638.

-. 1954. Caryologia, Vol. suppl. 1954: 1150-1152.

- 1955. Osterr. Bot. Zeitschr. 102: 195-234.

. 1956. Ber. Bayer. Bot. Ges. 31: 5-12.

Ellis, J. R. 1958. Proc. X Int. Congr. Genet. II: 74.

ERLANDSSON, S. 1942. Acta Hort. Berg. 13: 117-148.

ㄴ. 1946. Sv. Bot. Tidskr. 40: 427-435.

FAGerLIND, F. 1934. Hereditas 19: 223-232.

- 1937. Acta Horti Berg. 11: 195-470.

Favarger, C. 1959. Ber. Schweiz. Bot. Ges. 69: 24-46.

FELFöLdY, L. 1947. Arch. Biol. Hung. II, 17: 101-103.

Fernandes, A. 1950. Agron. Lusit. 12: 551-600.

- J. Garcia and R. Fernandes. 1948. Mem. Soc. Brot. 4: 1-89.

Fothergill, P. G. 1936. Proc. Univ. Durham. Philos. Soc. 9: 205-216.

-. 1944. New Phytol. 43: 23-35.

Gadella, T. W. J. 1962. Froceedings Roy. Neth. Acad. Scienc. Ser. C, 65, 3: 269-278.

1963. Act. Bot. Neerl. 12: (in press).

Gagniev, A. and R. Linder. 1955. Jard. Bot. Strasbourg. Index Seminum 1955: $19-22$.

GertuER, L. 1936. Jahrb. wiss. Bot. 83: 707-724.

Gentschefr, G. 1937. Planta 27: 165-195.

Gershox, A. 1928. Vermont Agric. Exp. Stat. Bull. 279: 1-18.

Goodway, K. M. 1955. Proc. Bot. Soc. Brit. Isles 1: 383.

1957. In Lousley, J. E. (ed.): Progress in the study of the British Flora, 1957: 116-118.

Gregory, W. G. 1941. Trans. Am. Phil. Soc. N.S. 31: 443-520.

Griesinger, R. 1937. Ber. Deutsch. Bot. Ges. 55: 556-571.

GuinocheT, M. 1942. Bull. Soc. Bot. Fr. 89: 70-75.

H. 1943. Rev. Cytol. Cytophysiol. Veg. 6: 209-220.

HAGERUP, O. 1938. Hereditas 24: 258-264.

HAGERUP, 1941. Bot. Tidsskr. 45: 385-395.

- 1944a. Hereditas 30: 152-160.

- 1944b. Dansk Bot. Ark. 11, 5: 1-26.

-1945. Dansk Vid. Selsk. Biol. Medd. 19, 11: 1-13.

-. 1947. Dansk Vid. Selsk. Biol. Medd. 20, 9: 1-22.

Hakansson, A. 1953. Bot. Not. 1953: 301-307.

Hamel, J. L. 1953. Rev. Cytol. Biol. Veg. 14: 113-313.

Hancock, B. L. 1942. New Phyt. 41: 70-79.

HaRA, H. 1954. VIII Congr. Int. Rapp. et Comm. 9 \& 10: 71-72.

and S. Kurosawa. 1956. Bot. Mag. Tokyo 69: 345-352.

Harada, I. 1948. Bot. Mag. Tokyo 61: 81-82.

- 1952. Jap. Journ. Genet. 27: 117-120.

. 1956. Cytologia 21 : 306-328.

Hasegawa, N. 1933. Bot. Mag. Tokyo 47: 901-903.

Hedberg, O. 1958. Sv. Bot. Tidskr. 52: 37-46.

HeGI, G. (1906/1931) Ill. Fl. Mittel-Europa V.4.

Hegnauer, R. and T. Meijers, 1958. Planta medica 6: 349-372.

HeItz, E. 1926. Zeitschr. Bot. 18: 625-681.

HeNDRICKs, A. J. 1957. Am. Midl. Nat. 58: 470-493.

Heslop-Harrison, J. 1948. Trans. Bot. Soc. Edinb. 35: 26-66. 
1951. Sv. Bot. Tidskr. 45: 608-635.

Hrss, H. 1953. Ber. Schweiz. Bot. Ges. 63: 267-270.

Heusser, C. 1938. Ber. Schweiz. Bot. Ges. 45: 562-605.

HocQueTte, W. 1922. C. R. Soc. Bot. Fr. 87: 1301-1303.

Hoffmann, K. M. 1929. Ber. Deutsch. Bot. Ges. 47: 321-326. 1930. Planta 10: 523-595.

Holmen, K. and P. KaAd. 1956. Bot. Tidsskr. 53: 35-48.

Holzer, K. 1952. Osterr. Bot. Zeitschr. 99: 118-155.

HoWARD, H. W. 1947. Nature, 159: 66.

HrUBY, K. 1932. Preslia 11: 40-44.

HubAc, J. M. 1961. Bull. Soc. Bot. France 108: 16-17.

HuBer, A. W. 1927. Jahrb. wiss. Bot. 66: 359-380.

Hutton, E. M. and J. W. Peak, 1954. Austr. Journ. Agr. Res. 5: 356-364.

Janaki-Ammal, E. K. and B. Saunders. 1952. Kew Bull. 1952: 539-542.

JARETZKY, R. 1932. Jahrb. wiss. Bot. 76: 485-527.

Jörgensen, C. A., T. Sörensen and M. WestergaAro. 1958. Dansk Vid. Selsk. Biol. Skr. 9, 4: 1-172.

Karpechenko, G. C. 1925. Bull. Appl. Bot. Genet. Plant Breed. 14: 271-279.

Kato, Y. 1951. Kromosomo 9-10: 342-347.

KECK, D. D. 1946. Proc. Calif. Acad. Sci. VIth Ser. 25: 421-468.

Kноsноо, T. N. and S. N. Sobti. 1958. Nature 181: 853-854.

KuIPHuis, E. 1962. Acta Bot. Neerl. 11: 90-92.

1962. Proc. Roy Neth. Acad. Scienc. Ser. C, 65, 3: 279-285.

1963. Acta Bot. Neerl. 12 (in press).

Kurrta, M. 1956. Jap. Journ. Genet. 31 : 330-333.

- 1957. Rep. Biol. Inst. Ehime Univ. 2: 1-8.

- 1958a. Rep. Biol. Inst. Ehime Univ. 5: 1-14.

1958b. Rep. Biol. Inst. Ehime Univ. 6: 9-16.

Langlet, O. F. J. 1927. Sv. Bot. Tidskr. 21: 1-17.

LARsen, K. 1954. Bot. Tidsskr. 50: $163-174$.

LARSEN, 1960. Dansk Vid. Selsk. Biol. Skr. 11, 3: 1-60.

LARTER, L. N. H. 1932. Jour. Genet. 26: 255-283.

Lawalree, A. 1952. Bull. Jard. Bot. Brux. 22: 193-200.

Lawrence, W. J. C. 1932. Genetica 13: 183-208.

Ledingham, G. F. 1957. Can. Journ. Bot. 35: 657-666.

Levitsky, G. A. 1940. Bot. Zhurn. 25: 292-296.

Linder, R. and J. BRun. 1956. Jard. Bot. Strasbourg. Index Seminum 1956: 28-34. Lohammar, G. 1931. Sv. Bot. Tidskr. 25: 495-499.

Löve, A. 1950. Bot. Not. 1950: 24-60.

. 1951. Caryologia 3: 263-284.

LOVE, A. and D. 1942a. Kungl. Fysiogr. Sällsk. Lund Forhandl. 12, 6: 58-76.

- 1942b. Bot. Not. 1942: 19-59.

- 1944. Ark. Bot. 31 A, 12: 1-23.

- 1948. Icel. Univ. Inst. Appl. Sci. Dept. Agr. Rep. B, 3: 1-131.

- 1956. Acta Hort. Gotob. 20: 65-291.

. 1961. Opera Botanica, suppl. Bot. Not. 5: 1-581.

Löve, D and J. P. BERnard. 1959. Sv. Bot. Tidskr. 53: 335-461.

Lövkvist, B. 1947. Hereditas 33: 421-422.

L. 1953. Proc. VII Int. Bot. Congr. (1950): 333-334.

-. 1956. Symb. Bot. Upsal. 14, 2: 1-131.

- 1957a. Bot. Not. 110: 237-250.

C. 1957b. Bot. Not. 110: 423-441.

MacMarion, B. 1936. Cellule 45: 209-262.

Manton, I. 1929. Ann. Bot. 46: 509-556.

Marchal, E. 1920. Mem. Acad. Roy. Belg. II, Cl. IV, 8: 1-108.

Marsden-Jones, E. M. and W. B. Turrill. 1935. Journ. Genet. 31: 363-378. Martin, R. W. and F. H. Smith. 1955. Bot. Gaz. 116: 243-249.

MatsuURA, H. and T. Suto. 1935. Journ. Fac. Sci. Hokkaido Imp. Univ. V, 5: 33-75. 
Maude, P. F. 1939. New Phytol. 38: 1-31.

M. 1940. New Phytol. 39: 17-32.

Meurman, O. 1925a. Soc. Sci. Fenn. Comm. Biol. II, $2: 1-4$. 1925b. Soc. Sci. Fenn. Comm. Biol. II, 3: 1-105.

MrYaJI, Y. 1927 . Bot. Mag. Tokyo 41: 568-569.

MookerjeA, A. 1956. Ann. Bot. Soc. Vanamo 29: 1-44.

Moore, R. J. 1958. Canad. Journ. Bot. 36: 547-559. , and D. R. Lindsay. 1953: Canad. Journ. Bot. 31: 152-163.

MoRI, M. 1957. Caryologia 9: 365-368.

MorIYA, A. and A. Kondo. 1950. Jap. Journ. Genet. 25: 131-134.

Morley, F. H. W., R. D. Brock and G. I. Davern. 1956. Austr. Journ. Biol. Sci. 9: 1-17.

Mulligan, G. A. 1957. Canad. Journ. Bot. 35: 779-789.

1958. Rhodora 60: 122-125.

Nakajima, G. 1936. Jap. Journ. Genet. 12: 211-218.

Negodi, G. 1937. Atti Soc. Nat. Mat. Modena $68: 9-11$.

Neves, J. de Barros. 1944. Diss. Univ. Coimbra 1944.

1950. Agron Lusit. 12: 601-610.

-. 1956a. Bol. Soc. Brot. 30: 141.

Palmaren, O. 1943. Bot. Not. 1943: 348-352.

Perry, B. A. 1943. Am. Journ. Bot. 30: 527-543.

PolyA, L. 1949. Acta Geobot. Hung. 6: 124-137.

1950. Ann. Biol. Univ. Debrecen 1: 46-56.

Poporp, A. 1935. Planta 24: 510-522.

PoucQues, M. L. DE. 1948. Bull. Soc. Sci. Nancy N.S. 7: 33-39.

P. 1949. Rev. Gen. Bot. 56: 1-27, 74-138, 172-188.

Priestley, C. A. 1953. Bot. Soc. Brit. Isles, Yearb. 1953: 63.

Pritchard, T. O. 1957. Journ. Ecol. 45: 965-973.

-. 1959. X Int. Bot. Congr. Vol. II, Abstr.: 311.

Protassenja, G. D. 1930. Arb. Bot. Lab. Centr. Moorversuchsstat. Minsk 1: 83-92.

Qúzzel, P. 1957. Encycl. Biogeogr. et Ecol. 10: 1-445.

RAO, Y. S. 1953. Proc. Natl. Inst. Sci. India 19: 563-583.

Renkomen, $K$. O. and E. Therman. 1952. Ann. Med. Exp. Fenn. 30: 327-328.

Richardson, M. M. 1933. Univ. Cal. Publ. Bot. 17: 51-60.

Rodrigues, J. E. DE. 1953. Diss. Univ. Coimbra 1953.

Rodriguez, R. L. 1949. Madrono 10: 95.

ROHWEDER, H. 1937. Planta 27: 501-549.

Roscoe, M. 1927. Bot. Gaz. 84: 307-316.

Roust, A. 1956. Ann. Bot. Soc. Vanamo 29, 2: 1-64.

R. 1961. Hereditas 47: 81-110.

Rozanova, M. A. 1940. Zhurn. Bot. SSSR 25: 304-308.

RuNQuIST, E. 1937. Hereditas 23: 279-286.

Rut 1938. Bot. Not. 1938: 235-243.

Rutland, J. P. 1941. New Phytol. 40: 210-214.

Ryka, C. 1954. Acta Soc. Bot. Polon. 23: 321-333.

Sakamura, T. 1914. Bot. Mag. Tokyo 28: 131-147.

SarkanY, S. and G. Baranyai. 1958. Acta Bot. Acad. Sci. Hung. 4: 311-350.

SATo, D. 1942. Jap. Journ. Bot. 12: 57-161.

ScheEl, M. 1931. Bot. Archiv. 32: 148-208.

Scheerer, H. 1940. Planta 30: 716-725.

SCHmid, A. 1961. Osterr. Bot. Zeitschr. 108: 20-88.

SCHÖFER, G. 1954. Planta 43: 537-565.

Schulz-Gaebel, H. H. 1930. Beitr. Biol. Pflanzen 18: 345-398.

Senjaninova, M. 1926. Zeitschr. Zellforsch. mikrosk. Anat. 3: 417-430.

. 1927. Zeitschr. Zellforsch. microsk. Anat. 5: 675-679.

SENN, H. A. 1938. Bibliogr. Genet. 12: 175-345.

Sharma, A. K. 1956. Caryologia 11: 93-130. 
Shimotomai, N. 1937a. Cytologia Fujii Jub. Vol.: 551-552.

- 1937b. Zeitschr. Vererb. 74: 30-33.

Shimoyama, S. 1959. Natl. Inst. Genet.( Japan) Ann. Rep. 9: 51-53.

Simoner, M. 1928. C. r. Soc. Biol. Paris 99: 1314-1316.

C. 1932. Bull. Biol. Fr. \& Belg. 105: 255-444.

Skalinska, M. 1945. Proc. Linn. Soc. London. B. 157: 4.

1946. Acta Soc. Bot. Polon. 17: 39-45.

-1947. Journ. Linn. Soc. London Bot. 53: 159-186.

- 1950a. Acta Soc. Bot. Polon. 20: 40-68.

-1950b. Bull. Int. Acad. Polon. Sci. Lettr. B: Sci. Nat. (1): 149-175.

—. 1954. Acta Soc. Bot. Polon. 23: 359-374.

Sraut 1958. Acta Biol. Cracov. Ser. Bot. 1: 45-54.

Skalinska, M., E. Banach-Pogan, H. Wcislo, et al. 1957. Acta Soc. Bot. Polon. 26: 215-246.

and R. Czapik. 1959. Acta Biol. Cracov., Ser. Bot. 1: 137-149.

$\longrightarrow$, M. Piotrowicz, et al. 1959. Acta Soc. Bot. Polon. 28: 487-529.

, M. Piotrowicz, A. Sokolowska-KulczYcka, et al. 1961. Acta Soc. Bot. Polon. 30: 463-489.

Sokolovskaja, A. P. 1958. Bot. Zhurn. 43: 1146-1155.

\begin{tabular}{l} 
- 1960. Vestnik Leningr. Univ. Ser. Biol. 4: no. $21: 42-58$. \\
\hline
\end{tabular} 418.

$\longrightarrow$, and $\longrightarrow$ 1941. Doklady Akad. SSSR. N.S. 32: 144-147.

Sorokin, H. 1924. Publ. Fac. Sci. Univ. Charles, Praha, 13: 1-15.

1927a. Am. Journ. Bot. 14: 76-84.

- $1927 \mathrm{~b}$. Genetics 12: 59-83.

—. 1927c. Am. Nat. 61: 571-574.

- 1927d. Am. Journ. Bot. 14: 565-581.

SPRUMonT, G. 1928. Cellule 38: 271-292.

StÄhlin, A. 1929. Wiss. Arch. Landwirtsch. 1: 330-398.

Staner, P. 1929. Cellule 39: 217-231.

Strey, M. 1931. Planta 14: 682-730.

Sugiura, T. 1938. Proc. Imp. Acad. Tokyo 14: 391-392.

. 1940. Cytologia 10: 324-334.

- 1942. Cytologia 13: 418-432.

Suomalainen, E. 1947. Ann. Acad. Sci. Fenn. A, IV, Biol. 13: 1-67.

SuzukA, O. 1950. Seiken Ziho 4: 57-58.

Sveschnikova, I. N. 1927. Bull. Appl. Bot. Genet. Pl. Breed. 17: 37-72.

- 1928. Zeitschr. Vererb. Suppl. 2: 1415-1421.

- 1929. Ann. Timirjasev Acad. 4: 1-22.

-_._. 1937. Biol. Zhurn. 6: 949-969.

Tahara, M. 1921. Journ. Coll. Sci. Tokyo Imp. Univ. 43, 7: 1-54.

Tarnavschi, I. T. 1948. Bull. Jard. Mus. Bot. Univ. Cluj 28 suppl.: 1-130.

TAteOKa, T. 1956a. Natl. Inst. Genet. (Japan) Ann. Rep. 6: 73-74.

. 1956b. Bot. Mag. Tokyo 69: 112-117.

Terby, J. 1922. Cellule 32: 197-226.

Therman-Suomalainen, E. 1949. Hereditas 35: 86-108.

Therman, E. 1953. Hereditas 38: 277-288.

Tischler, G. 1931. Tab. Biol. 7: 109-226.

TISCHLER, 1935. Tab. Biol. 11 : 281-304.

- 1937. Tab. Biol. 12: 57-115.

1950. Die Chromosomenzahlen der Gefässpflanzen Mitteleuropas. 's-Gravenhage.

Tond, B. 1942. Vasculum 27: 26-27.

Tomaszewski, A. 1959. Acta Soc. Bot. Polon. 28: 695-704.

Tschermak-Woess, E. Österr. Bot. Zeitschr. 95: 270-276.

Turesson, G. 1938. Ann. Roy. Agr. Coll. Sweden 5: 405-416.

T, and Turesson, B. 1960. Hereditas 46: 717-736.

Tuschnjakova, M. 1929. Planta 7: 29-44.

Valentine, D. H. 1941. New Phytol. 40: 189-209. 
1949. In Wilmott, A.J. (ed.): British Flowering Plants and Modern Systematic Methods, 1949: 48-53.

1950. New Phytol. 49: 193-212.

Walther, E. 1949. Mitt. Thür. Bot. Ges. Beih. 1: 1-108.

WANSCHER, J. H. 1931. Hereditas 15: 179-184.

1932. Bot. Tidsskr. 42: 49-58.

WeIjer, J. 1952. Genetica 26: 1-32.

West, G. 1930. Am. Journ. Bot. 44: 87-110.

WEWETZER, H. 1956. Mikrokosmos 46: 33-38.

Wexelsen, H. 1928. Univ. Calif. Publ. Agr. Sci. 12: 355-376.

Whitaker, T. W. 1934. Journ. Arn. Arb. 15: 135-143.

WHYTE, R. O. 1929. Journ. Genet. 21: 183-191.

WINGe, O. 1917. C. r. Trav. Lab. Carlsberg 13: 131-275.

WIPF, L. 1939. Bot. Gaz. 101: 51-67.

Wulfe, H. D. 1937a. Planta 26: 275-290.

. 1937b. Jahrb. wiss. Bot. 84: 812-840.

- 1937c. Ber. Deutsch. Bot. Ges. 55: 262-269.

- 1938. Ber. Deutsch. Bot. Ges. 56: 247-254.

- 1939. Ber. Deutsch. Bot. Ges. 57: 84-91. 1950. Ber. Deutsch. Bot. Ges. 63: 64-70.

Yates, J. J. and N. H. L. Brittan. 1952. Austr. Journ. Agr Res. 3: 300-304. 